\title{
Estimation of initial rate from discontinuous progress data
}

Accepted manuscript in Biocatalysis and Biotransformation (with embedded figures)

Peter J Halling

WestCHEM, Department of Pure \& Applied Chemistry, University of Strathclyde, Glasgow G1 1XL, UK.

Peter Halling, Dept P \& A Chemistry, University of Strathclyde, Glasgow G1 1XL, UK.

+44 141548 2683; p.j.halling@strath.ac.uk

ORCID: 0000-0001-5077-4088; www.linkedin.com/in/peter-halling-85116b17

Acknowledgements. I thank a former undergraduate project student Adam Evans who made some initial studies on this topic, including finding the paper of Poor (1968). He also made some interesting findings on human judgment of initial rates, a topic not covered in the present paper. I am grateful to several colleagues who gave details of how they currently would estimate initial rates from discontinuous data (Milan Polakovic, Wolf-Deiter Fessner, Peter Both and especially Anders Magnusson, who gave extensive information).

\begin{abstract}
When using discontinuous assay of reactions, initial rates are often estimated from a limited number of time points. There has been no detailed study of how best to do this. In this work, time courses were simulated by different theoretical equations (including strong product inhibition, first order, Michaelis-Menten and truly linear), but with random error addition to each data point. Various methods were tested to fit an initial rate to the data, and the result compared with the known "true" value. Fitting a simple quadratic generally gives initial rates as accurate as any other curve, and is better than a linear fit if there are about 8 or more time points. For fewer points a linear fit gives less variable and often more accurate rates. The absolute contribution to data point error has a major impact on rate accuracy, and often dominates that due to curvature, so that sampling to at least $10 \%$ conversion is preferred. The accuracy of a linear fit can be improved by methods that reject some later points based on curvature tests. Awareness of these effects can help avoid rate inaccuracies of $10 \%$ or more due to poor methods of data analysis.
\end{abstract}

Keywords: initial rate, simulation, discontinuous, fitting, algorithms 


\section{INTRODUCTION}

When measuring the progress of an enzymatic or other reaction, it is common to estimate an initial rate, as the slope of a linear phase in the early stages. The initial rate may be used just to summarise the observations, but is often the basis for further kinetic analysis. Where the reaction progress is effectively continuously recorded, such as by a spectroscopic method, identification of an initial linear phase is relatively trivial. (Cornish-Bowden (2012) suggests a method to ensure that the correct straight line has been drawn, p 87.) But often analysis of reaction progress requires discontinuous individual measurements, usually on samples removed from the reaction mixture at different times. In such cases there will usually be a limited number of data points (often less than 10), and some of these may be beyond the initial linear period. Of course each data point will be subject to experimental error, so none of them will lie precisely on a straight line. Such discontinuous assays are particularly common in applied biocatalysis, where a preparative reaction must be followed, rather than one selected for ease of monitoring. Even with some potentially continuous methods, like spectrophotometry, automated multiplexing (e.g. with a plate reader) may render data effectively discontinuous, although perhaps with less error than when sample handling is involved.

With discontinuous measurement it becomes more difficult to estimate initial rate, and different methods may give significantly different answers. Surprisingly, there seems to have been little detailed study comparing methods to use in such cases. Poor (1968) suggested a method based on the Newton-Gregory interpolation equation. This method does not seem to have been much used, but is considered in more detail in Supporting Information. In the authoritative book on enzyme kinetics, Cornish-Bowden (2012) highlights how a linear fit to discontinuous points can mislead ( $p$ 87) and suggests that an integrated rate equation may be a better option. Liu et al (2009) present an algorithm to switch between a linear fit and the use of the integrated MichaelisMenten equation for an enzyme believed to follow these kinetics. They offer rules to select the number of points to include in a linear fit, but without discussion of their basis. Similarly they give a test to switch to the integrated equation, based on a single data point. Their general approach of simulating data and testing fits to them is that pursued in the current paper. Pinto et al (2019) propose a linearization of the integrated Michaelis-Menten equation to test when various interferences may be giving erroneous initial rate estimates. The program "inrate" in the package Simfit (http://www.simfit.org.uk) by W G Bardsley offers several nonlinear equations that can be fitted to data, giving then a zero time slope. The documentation (w_manual.pdf) suggests an initial trial with a quadratic fit, followed by manual inspection to judge whether this is a sensible option.

There is an extensive literature on fitting curved progress curves to integrated forms of rate equations in order to determine kinetic constants (Duggleby, 1994; Rakels et al, 1994; Goudar et al, 1999; Tang \& Leyh, 2010; Golicnik, 2014). This is a quite distinct activity, where the objective is to estimate multiple kinetic constants, using data points extending well into clearly curved sections of the progress graph. The current paper deals with cases where the curvature is relatively small, and the objective is purely to estimate the initial rate.

From discussions with others in the field I am aware of the following possible approaches:

A. Judging where curvature starts and fitting a straight line to points before that

B. Using experience to select points in the linear region (e.g. up to a conversion limit) and fitting a straight line

C. Fitting a non-linear equation and calculating zero time slope from this 
D. Completely human estimation of where an initial rate straight line should be drawn

All these methods require some subjective opinion from the person employing them. (In the case of $\mathrm{C}$ this comes in selection of the equation to be used.)

To give an impression of typical practice, 12 recent papers reporting initial rates from discontinuous assays of enzyme activity were selected, essentially randomly. They had been published in respected journals (Analytical Biochemistry, Biocatalysis \& Biotransformation, Biochemical Engineering Journal, Biochemistry, Biotechnology \& Bioengineering, Catalysis Science \& Technology, Chemical Engineering Science, Journal of Chemical Technology \& Biotechnology, Proceedings of the National Academy of Sciences USA and RSC Advances). Full details have been made available to editors and referees, but are not given here because the aim is not to criticise individual authors, but rather to illustrate accepted practice in the field. I am fairly sure that none of my own papers reporting discontinuous assays have given full details of how initial rates were obtained from time course data.

Of these 12 papers, 5 gave no information on how initial rates were estimated from data points, and 3 more just noted a maximum conversion up to which points were used (10\%, $14 \%$ and $25 \%)$. One further paper reported a straight line fit without further details, while another implied rates were calculated from two selected data points. Two final papers fitted expected non-linear equations to the data and calculated initial rates analytically (Soda et al, 2017; Varga et al, 2017).

Seven papers that reported initial rates for non-enzymatic chemical reactions using discontinuous analysis were also examined (in Chemical Engineering Journal (2), Journal of the American Chemical Society (3), Nature Communications and Physical Chemistry Chemical Physics). My comments here are less reliable because of limited understanding of what methods may be taken as standard in this community. Only one of these papers gave no information on how initial rate was estimated. Three showed example progress curves with fitted straight lines, but some of the time courses looked non-linear. One more paper said straight lines were fitted, without examples. One fitted $4^{\text {th }}$ or $5^{\text {th }}$ order polynomials to estimate a zero time initial rate, without justification, and one final paper fitted a theoretical curve believed to reflect the kinetics.

In the present study, the effectiveness of different methods to estimate initial rate have been tested by using simulated data (including errors). Then the initial rates found by various models and algorithms can be compared with the "true" values used in simulation. This allows some clear general recommendations to be made.

\section{Theory and equations}

In all the following we assume that the reaction is the simple conversion of one molecule of starting material (substrate) into one of product, and that product concentration $\mathrm{P}$ is zero at zero time. The starting material concentration at zero time is $\mathrm{S}_{0}$. These equations are used in the current paper both in simulating the values of $P$ at various times, and in fitting the resulting simulated data (after addition of errors). 


\subsection{Units and dimensions}

The behaviour is shown in a way independent of the scales of concentration or time that might be found in a particular experiment. The equations for reaction progress are usually written in terms of concentrations, times and kinetic parameters, all of which have units. Indeed, the simulations used in this study were run with numbers having notional units of $\mathrm{mM}$ and min. But they can easily be transformed to dimensionless values that capture universal behaviour. Instead of using product concentration, this can be divided by the initial substrate concentration $\left(\mathrm{S}_{0}\right)$ to give the dimensionless value of conversion, c. Similarly an initial rate with units of concentration change per unit time can be divided by the initial substrate concentration to give a value $v_{0} / S_{0}$ with dimensions of time ${ }^{-1}$ (being in fact change in conversion per unit time). This value is given the symbol $\mathrm{v}_{0}{ }^{\prime}$ below. Although it still has dimensions, it could be multiplied by a characteristic time for any reaction to give a dimensionless value of 1 . In several models, the extent of curvature is controlled by an adjustable parameter, which can have dimensions ( time $^{-1}$ for $k_{d}$, time $^{-2}$ for $a^{\prime}$ in the quadratic model). But these parameters can also be multiplied by the same characteristic time to give dimensionless values that describe a universal degree of curvature. This is shown mathematically for the case of the quadratic model below. Hence the progress curve for any dataset or fitted line can be transformed to a dimensionless example that covers all possible cases. This corresponds to changing the scale of the time axis on a progress curve of conversion - with an appropriate time scale, the conversions reached will be identical for a given value of a dimensionless curvature-controlling parameter.

In weighted least squares fitting, it is also necessary to make an assumption about the relative contributions to errors in the data points. The fractional contribution to error is clearly a dimensionless quantity, having the same value whether seen as a fraction of $\mathrm{P}$ or of $\mathrm{c}$. The fixed or absolute contribution to the datapoint error has units if used with P. But in this paper it is presented as a dimensionless fraction of $\mathrm{S}_{0}$. By working in terms of conversion and other dimensionless values, we can see behaviour independent of the particular concentrations and times relevant to a particular experiment.

In summary, in this paper, unless units are given, all concentrations are given as a dimensionless fraction of the initial substrate concentration $\mathrm{S}_{0}$. This includes the absolute error that would normally have concentration units.

\subsection{Models}

First order.

The simple first order kinetic model integrates to give

$$
P=S_{0}\left(1-e^{-k t}\right)
$$

which can also be written in terms of conversion c as

$$
c=\left(1-e^{-k t}\right)
$$

The analytical initial rate is $k . S_{0}$, or after removing the dimensions of concentration, we get $v_{0}{ }^{\prime}=k$. 
Second order.

The second order model integrates to give

$$
P=\frac{k \cdot S_{0}^{2} \cdot t}{1+k \cdot S_{0} \cdot t}
$$

or

$$
c=\frac{k \cdot S_{0} \cdot t}{1+k \cdot S_{0} \cdot t}
$$

The analytical initial rate is $\mathrm{k} \cdot \mathrm{S}_{0}{ }^{2}$, or after removing the dimensions of concentration, $\mathrm{v}_{0}{ }^{\prime}=\mathrm{k} . \mathrm{S}_{0}$.

Hence

$$
c=\frac{v_{0}^{\prime} \cdot t}{1+v_{0}^{\prime} \cdot t}
$$

Deactivating catalyst.

Where the enzyme or other catalyst is deactivating with first order kinetics, but the still active catalyst works at a constant rate throughout, integration of the rate equations gives:

$$
P=\frac{v_{0}}{k_{d}}\left(1-e^{-k_{d} \cdot t}\right)
$$

which can be transformed to

$$
c=\frac{v_{0}^{\prime}}{k_{d}}\left(1-e^{-k_{d} \cdot t}\right)
$$

The form is related to that for the first order model, but in this case the adjustable parameter $k_{d}$ will control the extent of curvature.

Michaelis-Menten model.

The equation is integrated to give:

$$
P-K_{M} \cdot \ln \left(\frac{S_{0}-P}{S_{0}}\right)=V \cdot t
$$

where $K_{M}$ and $V$ are the parameters of this model. This equation has been used quite extensively for fitting to whole progress curves. However, the equation requires numerical solution for $\mathrm{P}$ at each time $\mathrm{t}$. There does exist a solution of the integrated equation that is explicit in P (Schnell \& Mendoza, 1997):

$$
P=S_{0}-K_{M} \cdot \omega\left[\frac{S_{0}}{K_{M}} \cdot \exp \left(\frac{S_{0}}{K_{M}}-\frac{V}{K_{M}} t\right)\right]
$$

but this requires the $\omega$ function which is only available in more specialist mathematical software. However, there is also an approximation to this solution (Schnell, personal communication) 


$$
P=\frac{S_{0}}{1+\frac{S_{0}}{K_{M}}} \frac{V}{K_{M}} t-\frac{S_{0}}{2\left(1+\frac{S_{0}}{K_{M}}\right)^{3}}\left(\frac{V}{K_{M}} t\right)^{2}-\frac{S_{0}\left(2 \frac{S_{0}}{K_{M}}-1\right)}{6\left(1+\frac{s_{0}}{K_{M}}\right)^{5}}\left(\frac{V}{K_{M}} t\right)^{3}+O\left(t^{4}\right)
$$

If we take a dimensionless starting material concentration $\beta=S_{0} / K_{M}$, and neglect the $4^{\text {th }}$ order terms, then we can write:

$$
\frac{P}{S_{0}}=c=\frac{1}{1+\beta} \frac{V}{K_{M}} t-\frac{1}{2(1+\beta)^{3}}\left(\frac{V}{K_{M}} t\right)^{2}-\frac{(2 \beta-1)}{6(1+\beta)^{5}}\left(\frac{V}{K_{M}} t\right)^{3}
$$

The initial rate $v_{0}^{\prime}$ is given by

$$
v_{0}^{\prime}=\frac{v_{0}}{S_{0}}=\frac{V}{K_{M}+S_{0}}
$$

So

$$
\frac{V}{K_{M}}=v_{0}^{\prime} \cdot\left(1+\frac{S_{0}}{K_{M}}\right)=v_{0}^{\prime} \cdot(1+\beta)
$$

Substitute in the above to give

$$
c=\frac{1}{1+\beta} \cdot v_{0}^{\prime}(1+\beta) t-\frac{1}{2(1+\beta)^{3}}\left(v_{0}^{\prime}(1+\beta)\right)^{2} t^{2}-\frac{(2 \beta-1)}{6(1+\beta)^{5}}\left(v_{0}^{\prime}(1+\beta)\right)^{3} t^{3}
$$

And cancel and simplify

$$
c=v_{0}^{\prime} \cdot t-\frac{1}{2(1+\beta)}\left(v_{0}^{\prime} \cdot t\right)^{2}-\frac{(2 \beta-1)}{6(1+\beta)^{2}}\left(v_{0}^{\prime} \cdot t\right)^{3}
$$

This equation was used in fitting simulated data sets, with the adjustable parameter $\beta$ controlling the extent of curvature. The equation was tested against numerical solutions of the $\mathrm{P}$ implicit integrated equation (8), and gave values that agreed to better than 1 part in 1000 over the range relevant for initial rate estimation.

\section{Quadratic model.}

From an empirical mathematical viewpoint, a very obvious way to describe a slightly curved progress is to express product concentration $\mathrm{P}$ as a quadratic in time $(\mathrm{t})$

$$
P=v_{0} \cdot t+a \cdot t^{2}
$$

where a negative value of parameter $a$ gives a rate declining with time, and $\mathrm{v}_{0}$ is initial rate. Removing the dimensions of concentration gives

$$
c=v_{0}^{\prime} \cdot t+a^{\prime} \cdot t^{2}
$$

where $a^{\prime}=a / \mathrm{S}_{0}$. As an example, in this case we show how the equation can be made completely dimensionless by defining a dimensionless time, $t^{\prime}=v_{0}{ }^{\prime} . t=v_{0} . t / S_{0}$. Hence $t=t^{\prime} . S_{0} / v_{0}$, and we get

$$
c=t^{\prime}+\frac{a \cdot S_{0}}{v_{0}^{2}} \cdot t^{\prime 2}
$$


which shows how the dimensionless coefficient of the square term can be calculated, as used in section 4.4.1.

Reverse Quadratic model.

Another model is quadratic in $\mathrm{P}$, and in this paper is referred to as Reverse Quadratic:

$$
P+a \cdot P^{2}=v_{0} \cdot t
$$

where positive values of $a$ give a rate slowing with time. After division through by $\mathrm{S}_{0}$, we obtain the form

$$
c+a^{\prime} \cdot c^{2}=v_{0}^{\prime} \cdot t
$$

To obtain P or c values the equation must be solved for each time, for example via the usual formula.

Equations with the form of the Reverse Quadratic and Quadratic models can be obtained as an approximation to solutions of other progress equations (see Supporting Information).

\section{Methods}

\subsection{Theoretical progress curves}

The tests used dimensioned values, but as explained above, the results can be presented in a universal dimensionless form, using division by initial substrate concentration and/or the true initial rate. All simulations used $\mathrm{S}_{0}=60 \mathrm{mM}$, and true initial rates of $0.7 \mathrm{mM} \mathrm{min}^{-1}$, or $0.75 \mathrm{mM} \mathrm{min}^{-1}$ for the highest curvature cases (datasets $\mathrm{P}$ and $\mathrm{D}$ ).

Five types of curve were calculated by integration of the equation for reversible Michaelis-Menten kinetics:

$$
\frac{d P}{d t}=\frac{V_{f}\left(S_{0}-P-\frac{P}{K_{e q}}\right)}{K_{S}+S_{0}-P+\frac{K_{S}}{K_{P}} \cdot P}
$$

where $\mathrm{P}$ is the product concentration at time $\mathrm{t}, \mathrm{S}_{0}$ is the initial substrate concentration, and the other symbols are kinetic constants. Numerical integration used the $5^{\text {th }}$ order Runge-Kutta algorithm with adaptive step size control and scaling, taken from Press et al, 1986, implemented as an Excel macro. The parameters used are shown in Table 1. By setting $K_{S}, K_{p}$ and $K_{\text {eq }}$ to very large values (the first two >> initial substrate concentration $S_{0}$ ), we get essentially first order kinetics $(F)$. Reducing $K_{S}$ to equal $S_{0}$, we get the Michaelis-Menten case (M). Reducing $\mathrm{K}_{\mathrm{s}}$ even further to $0.1^{*} \mathrm{~S}_{0}$ would give almost linear progress on its own, but using with it $\mathrm{K}_{\text {eq }}=1$ gives the equilibrium dominated case $(E)$. With again $K_{S}=0.1 * S_{0}$, but $K_{P}=S_{0} / 30$ gives the strong product inhibition case $(P)$, while $K_{P}=S_{0} / 12$ gives weaker product inhibition $(W)$. 
Table 1. Parameters for generation of "true" progress curves.

\begin{tabular}{|l|l|l|l|l|l|}
\hline Dataset type & $P$ & $M$ & $E$ & $F$ & W \\
\hline $\mathrm{V}_{\mathrm{f}}\left(\mathrm{mM} \mathrm{min}^{-1}\right)$ & 0.825 & 1.4 & 0.77 & 70.7 & 0.77 \\
\hline $\mathrm{K}_{\mathrm{s}}(\mathrm{mM})$ & 6 & 60 & 6 & 6000 & 6 \\
\hline $\mathrm{K}_{\mathrm{p}}(\mathrm{mM})$ & 2 & $10^{6}$ & $10^{6}$ & $10^{6}$ & 5 \\
\hline $\mathrm{K}_{\mathrm{eq}}$ & $10^{6}$ & $10^{6}$ & 1 & $10^{6}$ & $10^{6}$ \\
\hline
\end{tabular}

For the enzyme deactivation case (D), simulation used

$$
P=\frac{v_{0}}{k_{d}}\left(1-e^{-k_{d} \cdot t}\right)
$$

with $\mathrm{v}_{0}=0.75 \mathrm{mM} \mathrm{min}^{-1}$ and $\mathrm{k}_{\mathrm{d}}=0.025 \mathrm{~min}^{-1}$. Finally for truly linear or zero order progress $(\mathrm{Z}), \mathrm{P}$ was simply 0.7 $\mathrm{mM} \mathrm{min}^{-1}$ times $\mathrm{t}$.

\subsection{Error addition}

To each time point from the theoretical progress, an error term was added, drawn randomly from a normal distribution with appropriate variance. The assumed standard deviation used to calculate the error term was made up of the sum of two possible components. A fractional component gave a standard deviation proportional to the magnitude of the data point. Commonly used weightings were 0.02 and 0.04 , corresponding to measurement errors of $2 \%$ and $4 \%$. An absolute component was the same for all data points, and is presented non-dimensionally by taking as a fraction of the initial substrate concentration (section 2.1). Commonly used dimensionless absolute components were 0.0017 and 0.0033 . So for example, in a set with lower mixed error, a datapoint at 0.1 conversion would be simulated to have an error of $0.0017+0.02 * 0.1=$ 0.0037 , equivalent to $3.7 \%$ of this datapoint value.

The algorithm to produce normal deviates (Press et al, 1986) will of course occasionally (1 in 20) produce a value further than 2 standard deviations from the mean. Such points might have a disproportionately large effect on the fitting process, so the simulation algorithm rejected such deviates and used the next error value instead. In a real experimental situation such points would often be identified as outliers and not used in fitting.

The simulations were run with a product concentration set at zero at zero time. With an absolute contribution to the error, this will sometimes produce a negative product concentration after error has been added. In such cases the simulated point was instead set to zero. In many real analytical methods very small concentrations will not be estimated but the instrument will report zero (e.g. if a chromatographic peak is too small to be detected by the integrator).

\subsection{Fitting to simulated time courses}

All fitting was done by using the built-in Excel Solver (with GRG Non-linear method) to minimise the sum of weighted square deviations of the data points, by varying the initial rate and one or two additional parameters. All models included a fitted offset parameter (which could be positive or negative) that was added to every product concentration on the fitted line or curve. Some initial trials were made of fits without such offset parameters, so fitted progress was forced to pass through the origin. With some time courses the resulting fit 
would be rather far from many of the data points, as it was constrained to get reasonably close to a data point at zero or small time. This was particularly the case if errors were assumed to be entirely fractional, and thus very small for these initial data points. In some models a second adjustable parameter controlled the extent of curvature. Fitting was automated using an Excel VBA macro (further details in Supporting Information).

In fitting by weighted least squares, an error estimate is required for every data point. This was calculated from two contributions, one absolute and one as a fraction of the conversion or product concentration at that point (the same two contributions as considered in simulation). For most cases, 3 different error assumptions were tested. A purely absolute error used a dimensionless value of 0.008 . A purely fractional error used a value of 0.1 . And a mixed error assumption had contributions of 0.004 dimensionless absolute and 0.05 fractional, so the two contributions became equal at a conversion of $0.08(8 \%)$.

If error is assumed to be totally fractional, an early time point with $\mathrm{P}=0$ will be calculated to have zero error, so that its weighted square deviation is undefined (division by zero). To avoid such numerical problems, if the error was calculated as zero (to machine accuracy), it was substituted by a tiny value, normally $10^{-6}$. This prevented numerical problems, but did not significantly affect the fit, which was still required to bring the line extremely close to this point.

For the Quadratic, Reverse Quadratic and Deactivation models (section 2.2), the parameter that controls the curvature can change sign to give a progress curve with rate increasing over time. In some cases the errors in the simulated data meant that such a curve gave a better fit to the data points. In almost all such cases the result was an initial rate estimate that was much too low (it would be even lower than obtained by fitting a straight line to all the data). So a constraint was added to the fitting routine to set zero as a limit for the value of the curvature parameter - so this was not permitted to be positive for the Quadratic model or negative for the other two. For the Deactivation model, and certain derivations of the Reverse Quadratic one, this simply prohibits physically impossible true values of the parameter. When one of these models was fitted with the limiting zero value of the curvature parameter, often the other two would be also, so all 3 gave the same initial rate as a straight line fit to the whole data set.

For the Michaelis-Menten model, the curvature parameter $\beta$ is defined as $[S]_{0} / K_{M}$. As $\beta$ approaches infinity, the result is a straight line, while for $\beta$ of zero, the progress is first order. For physically meaningless negative values of $\beta$, the equation gives at first curvature greater than for the first order model (for $\beta<-0.6$ or so, the shape is no longer a simple curve). Where the underlying progress really was more curved, allowing a negative value of $\beta$ gave a better fit to the data and usually a more accurate initial rate estimate. So the values of $\beta$ were not constrained, and for many data sets the model is better seen as an empirical equation rather than one underpinned by a kinetic model.

Statistical significance of differences in means was estimated using Welch's modified (more stringent) twotailed $\mathrm{t}$ test, while $\mathrm{F}$ test was used to compare standard deviations.

\subsection{Excel files available}

Supporting Information gives links to Excel files containing: a) all the VBA code used in simulating data, and in fitting lines or curves; b) all the simulated data sets generated and fitted; c) all the parameters for the individual fits obtained, and various analyses of them. 


\section{Results and Discussion}

\subsection{General approach adopted}

In order to evaluate how various methods performed, datasets were simulated by computation. This meant that the true initial rates were known (unlike in real experiments), and could be compared with those found from the simulated datasets. First, a theoretical error-free progress curve was calculated, using an appropriate equation. As well as truly linear progress, examples with visible but fairly small curvature were considered. Then an error term was added to each data point, drawn randomly from a normal distribution with appropriate standard deviation. Each resulting time course was then analysed by various fitting algorithms under test, to see what initial rate was obtained. Normally a dataset of 20 time courses all with the same underlying errorfree progress were analysed, to smooth out effects of randomness in error allocation. As shown below, this was enough to make larger effects statistically significant.

\subsubsection{Error considerations}

Two contributions to random error in any measurement can be distinguished. There may be an absolute or constant contribution, independent of the size of the measured value. There may also be a fractional or proportional contribution, which gets larger as the measured value rises. In many experiments both contributions may be present. Wording is commonly seen suggesting that one may dominate, such as when errors in concentration measurements are reported as " $2 \%$ " (fractional) or " $0.1 \mathrm{mM}$ " (absolute). The simulations used in this study included cases where errors were purely absolute, purely fractional or mixed. The absolute contribution would normally have units, but to make these simulations general it is sensibly treated non-dimensionally, as a fraction of initial substrate concentration ("the dimensionless absolute contribution", see section 2.1). The parameters controlling these contributions are shown for each case presented below.

Lines or curves were then fitted to the simulated data in order to estimate the initial rate, and compare the value found with that used in the original simulation. Fitting was always based on minimising the sum of weighted square deviations: take the difference between the fitted line and the point, divide by the expected error in that point, then square. This clearly requires error estimates to be assigned to each point. One approach tested here is to assign the same error estimate to every point, i.e. to assume error is entirely absolute: this will give a simple sum of squares fit. Another approach tested was to assume that the error is entirely fractional. Most realistic is to use both an absolute and a fractional component in the error estimates. In these tests, this mixed error assumption used contributions that become equal at a conversion of $0.08(8 \%)$, with absolute error dominating at lower conversion and fractional error the main one at higher conversion. Note that the fit obtained is not affected by the assumed size of the individual error components, just their relative magnitude. If both are increased by the same factor, all weighted square deviations become smaller, but the position and parameters of the fitted line are not affected.

All models also included an adjustable offset added to all product concentrations, so that fitted lines and curves did not have to pass exactly through the origin. The offset was always fitted as a small value, but could be positive or negative. 


\subsubsection{Presentation of fitting results}

All results are presented on a dimensionless basis to demonstrate universal behaviour (see further details in section 2.1.). Reaction progress is measured in terms of conversion, the fraction of initial substrate concentration, and the dimensionless absolute error contribution is also a fraction of [S]o. Fitted rates found are presented as a fraction of the "true" value obtained as the derivative of the theoretical model at zero time: hence the "correct" initial rate is always 1. Standard deviations of fitted rates, and their root mean square (RMS) difference from the correct value, are normalised in the same way. Hence the results presented here can be translated to any particular experiment by changing back from dimensionless values to those appropriate for the conditions chosen.

\subsection{Linear fit to initial points}

\subsubsection{Data sets used}

Data sets were simulated for a reaction following Michaelis-Menten kinetics with the initial substrate concentration equal to $\mathrm{K}_{\mathrm{M}}$. For conversion up to $0.1(10 \%)$, this meant modest but visible curvature (in the absence of error), see Fig. 1. Each data point value was then adjusted by an error term, which could have both a fractional or proportional component and an absolute component. Examples of resulting data sets are shown in Fig. 1. 


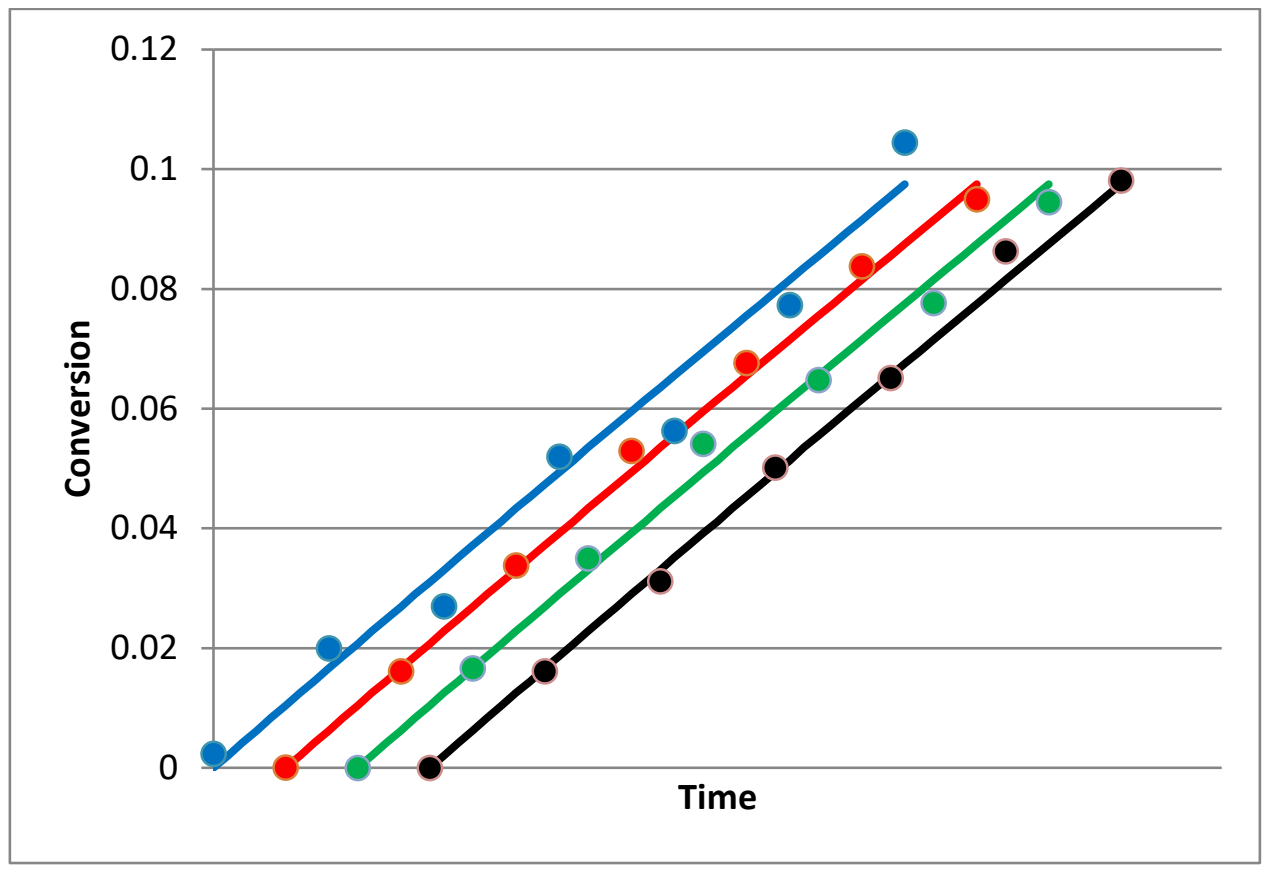

Figure 1. Example data sets simulated. The lines show the theoretical progress for the model used - MichaelisMenten with initial substrate concentration equal to $\mathrm{K}_{\mathrm{M}}$. The lines are slightly curved, although this is only evident on close examination. They are offset in time for display purposes. Example data points simulated are shown with each line (in each case these are for the first of 20 time courses simulated). From left to right these are for high mixed error ( $4 \%$ fractional and dimensionless absolute of 0.0033 ), low mixed error (half these values), pure absolute error (0.0033) and pure fractional error (4\%).

\subsubsection{Effect of number of data points}

Errors in the progress data points will always tend to cause variability in the initial rate estimates. Intuitively we might expect that increasing the number of data points would strongly reduce this variability. However, this effect actually seems to be quite weak (Fig. 2). In most cases there seems little to gain by having more than 5 data points, and even 4 data points usually gives a similar performance. Only in the case of pure absolute error, the initial rate seems to be more seriously underestimated with fewer data points (although this is not statistically significant, $p>0.1$ ). However, as might be expected, with only 4 points an unusually large error in one of them can make a particular initial rate estimate very poor. Hence further analysis focusses on the use of 5 data points. The standard deviations of fitted rates are rather similar for pure absolute error and high mixed error. These have the same size of absolute error component. This component is lower in the case of low mixed error, and zero in the case of pure fractional error, with corresponding reductions in standard deviation ( $p$ mostly $<0.05$, often $<0.001$ ). It appears that the absolute error component is critical in affecting initial rate estimates, as might be expected. 

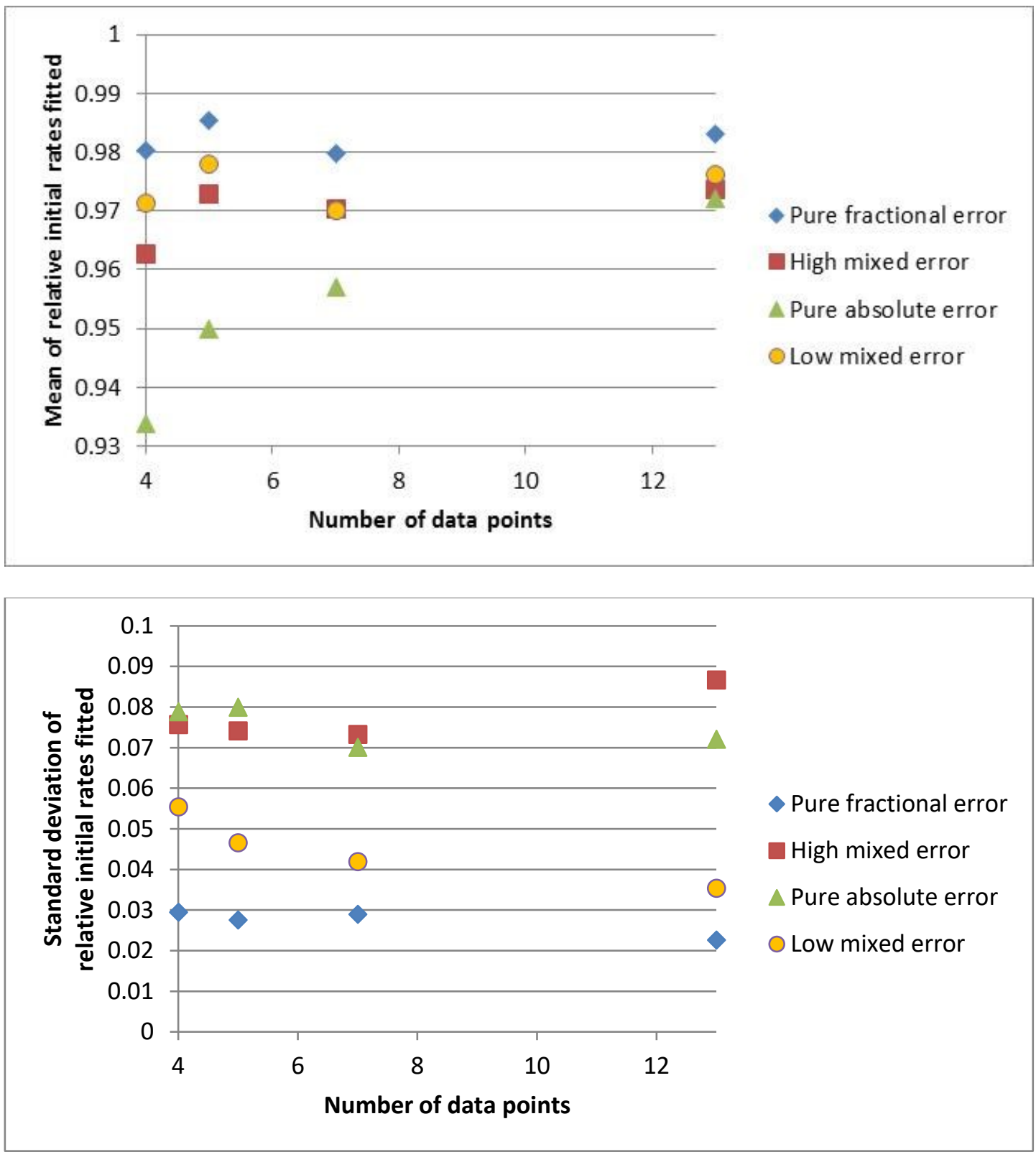

Fig 2. Effect of number of data points on fitted rates. A progress curve was calculated for a reaction following Michaelis-Menten kinetics with initial substrate concentration equal to $K_{M}$, and conversion up to 0.05 . Points were simulated by adding random error terms: the dimensionless absolute error contribution was 0.0033 for pure absolute and for high mixed error; the fractional error contribution was 0.04 for pure fractional and high mixed error; low mixed error used half these values. A total of 13 equally spaced points were simulated for each of 20 reactions, and other data sets were generated by selecting the zero time point, followed by every $2^{\text {nd }}$ point ( 7 total), every 3 rd point ( 5 total), or every $4^{\text {th }}$ point ( 4 total). A relative rate of 1 corresponds to the "correct" value, as determined from the analytical solution of the equation used in data simulation. Straight lines were fitted using the mixed error assumption. 


\subsubsection{Effect of size of error and maximal conversion}

As already apparent from the last section, the likely uncertainty in the initial rate estimate is highly dependent on the fixed absolute contribution to the error in the data points. This error contribution will normally dominate at the lowest conversion initial points, even when a fractional error contribution becomes large at higher product concentrations. Figure 3 shows how this error affects the accuracy and variability of the initial rate estimates. If points are restricted to conversions of less than 0.025 , the error in individual data points makes initial rate estimates vary very widely, unless the absolute error contribution is tiny. Most differences in standard deviation shown are significant (many $p<0.001$ ). The mean initial rate estimate also falls to more than $10 \%$ below the true value for large data point errors $(p<0.05)$. If the points are instead taken up to a conversion of 0.05 , the variation in initial rate estimates is reduced $(p<0.01)$. This variation is reduced even more with points up to 0.1 conversion $(p<0.01)$, although as expected the mean of initial rate estimates is rather lower ( $p<0.01$ for lower error cases), due to some curvature in the progress curves. This highlights the importance of minimising the absolute error contribution in real experiments. It may help the reader to show what these dimensionless absolute error contributions might mean in practice. The lowest value on the graphs, at about 0.0004 , would correspond to an error of $0.04 \mathrm{mM}$ in product concentration for a reaction with $0.1 \mathrm{M}$ starting material.

In contrast, the effect of the fractional error component was weak. For a fixed dimensionless absolute error component of 0.0033 , varying fractional error from 0 to 0.04 caused the standard deviation of fitted rates to increase by $50 \%$ at most (supporting information).

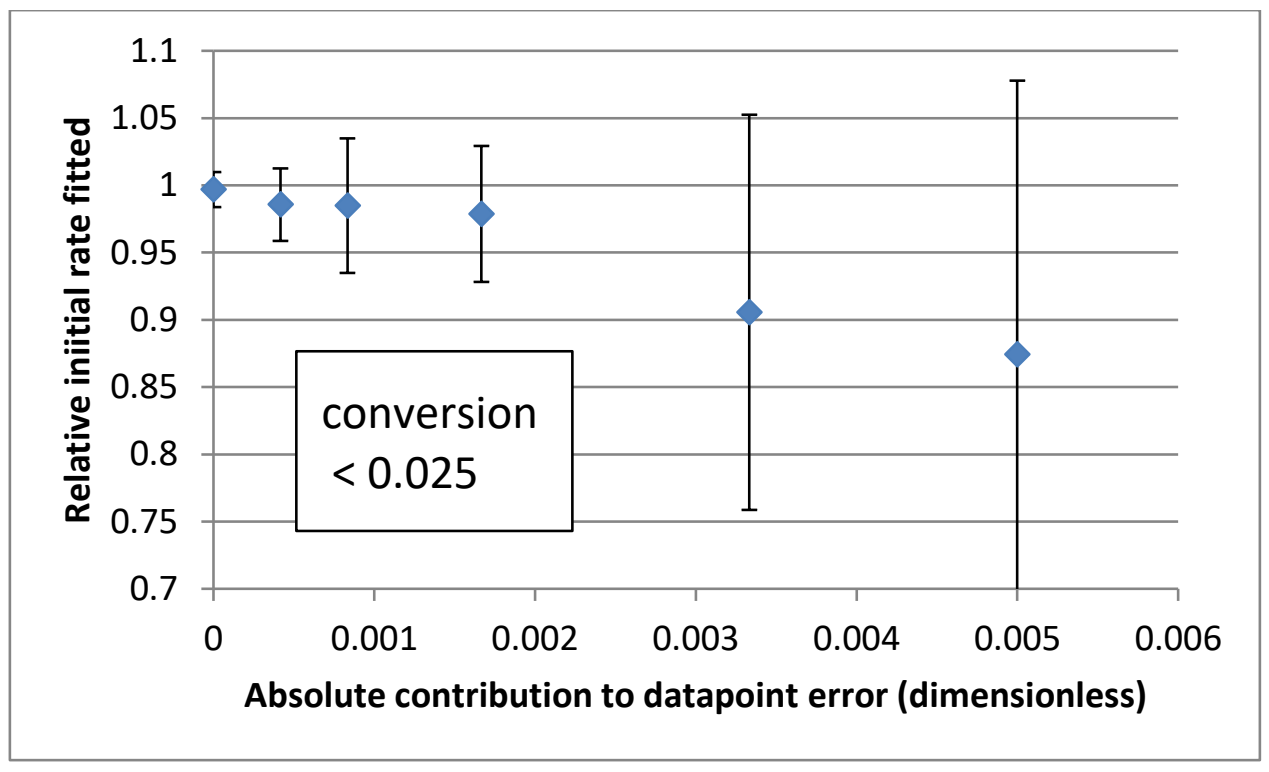



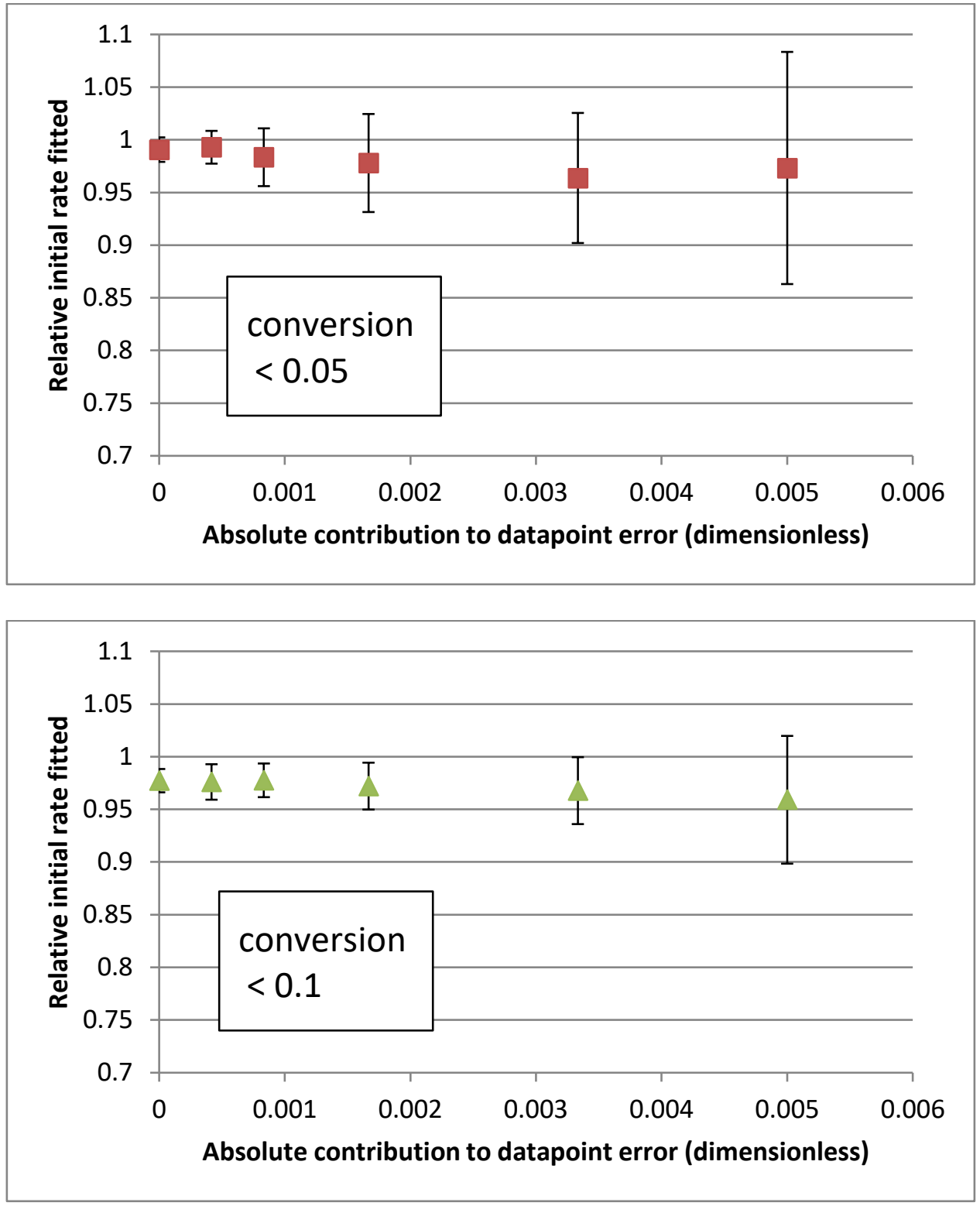

Fig. 3. Effects on initial rate estimates of absolute error magnitude and maximal conversion. A progress curve was calculated for a reaction following Michaelis-Menten kinetics with initial substrate concentration equal to $\mathrm{K}_{\mathrm{M}}$. Five data points were selected evenly spaced up to the conversion shown. Twenty progress curves were simulated with random error terms added, with a fractional contribution of 0.02 , and the absolute value shown. Straight lines were fitted using the mixed error assumption. A relative rate of 1 corresponds to the "correct" value, as determined from the analytical solution of the equation used in data simulation. 


\subsubsection{Error assumption in fitting}

The results above were based on fitting using the assumption of mixed error (with both absolute and fractional components). The effect of assuming pure fractional or pure absolute error was also investigated, and can be illustrated by examining datasets simulated using four different error conditions: pure absolute error with a dimensionless value of 0.0033; pure fractional error of 0.04; high mixed error with both these contributions; or low mixed error with both halved. Analysis considered averages for groups of 20 time courses with the same number of data points and maximum conversion. There were 12 such groups for each type of simulation error, so 48 in total, containing 960 time courses.

For datasets simulated on the basis of pure fractional error, and fitted with this assumption, the initial rates found were usually slightly higher (more accurate) than those fitted assuming mixed error. Similarly their standard deviations were slightly lower. However, for the other 3 error conditions used in simulation, assuming pure fractional error during fitting gave poor results. For all 36 such datasets, the mean initial rates were lower (less accurate) than for fitting based on mixed error, often much lower. And for 35 of these datasets, the fractional error assumption gave a higher standard deviation, typically about double. This all happened because with many time courses the line fitted assuming pure fractional error was very different from that for the other error assumptions. The line would be forced to get close to 2 or 3 initial points at low conversion, which would otherwise have very large weighted square deviations, because they were assumed to have very small errors. The other error assumptions would leave the line further from these points, if that gave a better fit to the full time course. This problem did not arise when the data had been simulated using purely fractional error, as the points at low conversion were then very close to the error-free line. It is however probably quite rare in a real experiment for there to be confidence that error is entirely fractional. Hence the assumption of purely fractional error in fitting is usually not a sensible approach.

The pure absolute and mixed error assumptions usually gave rather similar fitted lines. Cases where the fitted initial rates differed by more than $5 \%$ were only found where the simulation included higher absolute error contributions, and even then there were only 15 examples out of 480 time courses. Of 36 datasets (each of 20 time courses) with pure fractional or mixed error used in simulation, in 34 cases the standard deviation of rates was lower if fitting was done with mixed error assumption: however the differences were not large. There was no clear trend in the accuracy of the initial rate estimates. For data simulated with pure absolute error, assuming absolute error in fitting gave better initial rate estimates in 11 out of 12 groups, although again differences were not large. The assumption of mixed error has been used in fitting for the examples in previous sections, although similar plots are found for fitting assuming pure absolute error.

In summary, it is normally advisable not to assume pure fractional error while fitting. The choice between pure absolute error and mixed error is less critical, but the latter is probably better unless there are good reasons to believe that the true error is (almost) purely absolute. 


\subsection{Fitting a curve to the data}

Instead of a straight line, a curve can be fitted to the data points, and its equation used to obtain the initial rate as a zero time derivative. There are many different mathematical equations that might be used to describe the curve. The equations tested in this study are given in section 2.2 , corresponding to the following plausible models:-

- First order kinetics, a common general case, and found for enzymes when [S] $\ll \mathrm{K}_{M}$ (model First).

- Second order kinetics, another standard case, although rarely true for enzymes (model Second).

- Michaelis-Menten kinetics. The full integrated form of the equation requires numerical solution or uncommon functions, but there is a good approximation (model M-M).

- Enzyme deactivating with first order kinetics (model Deact).

- A simple quadratic equation in time, a very common way to describe a curve, but with no particular theoretical justification in this case (model Quad).

- An alternative equation quadratic in product concentration or conversion. This is actually an approximation to some more complicated theoretical models (see Supporting Information) (model Rev Quad).

For comparison, a linear fit for points up to a conversion of 0.1 was also used with the data (model Linear). With some of the models presented, it was found to be sensible to apply constraints on the sign of parameter values, as detailed in section 3.3.

With the use of different models for fitting, it was also appropriate to test different kinetic equations in the simulation of test data sets. The following were used:

- First order kinetics (datasets F)

- Michaelis-Menten kinetics for an initial substrate concentration equal to $K_{M}$ (datasets $M$ )

- Reversible Michaelis-Menten kinetics dominated by product inhibition (datasets P and W, with relatively high and weak curvature respectively)

- Reversible Michaelis-Menten kinetics dominated by approach to chemical equilibrium (datasets E)

- First order deactivation of the enzyme, but no other effect on its activity (datasets D)

- Zero order kinetics, completely linear (datasets Z).

In the non-linear cases parameters were selected such that progress curves showed fairly small but noticeable deviation from linearity for conversions up to around 0.2 (see Fig. 4). After random errors had been added to the data points, the curvature was often not immediately clear to the eye. As can be seen, the shapes of the curves are in fact not very different in this relatively early phase of the reaction. As will become clear, the bigger difference is probably the rather greater degree of curvature with the particular parameter choices for datasets $P$ and $D$. 


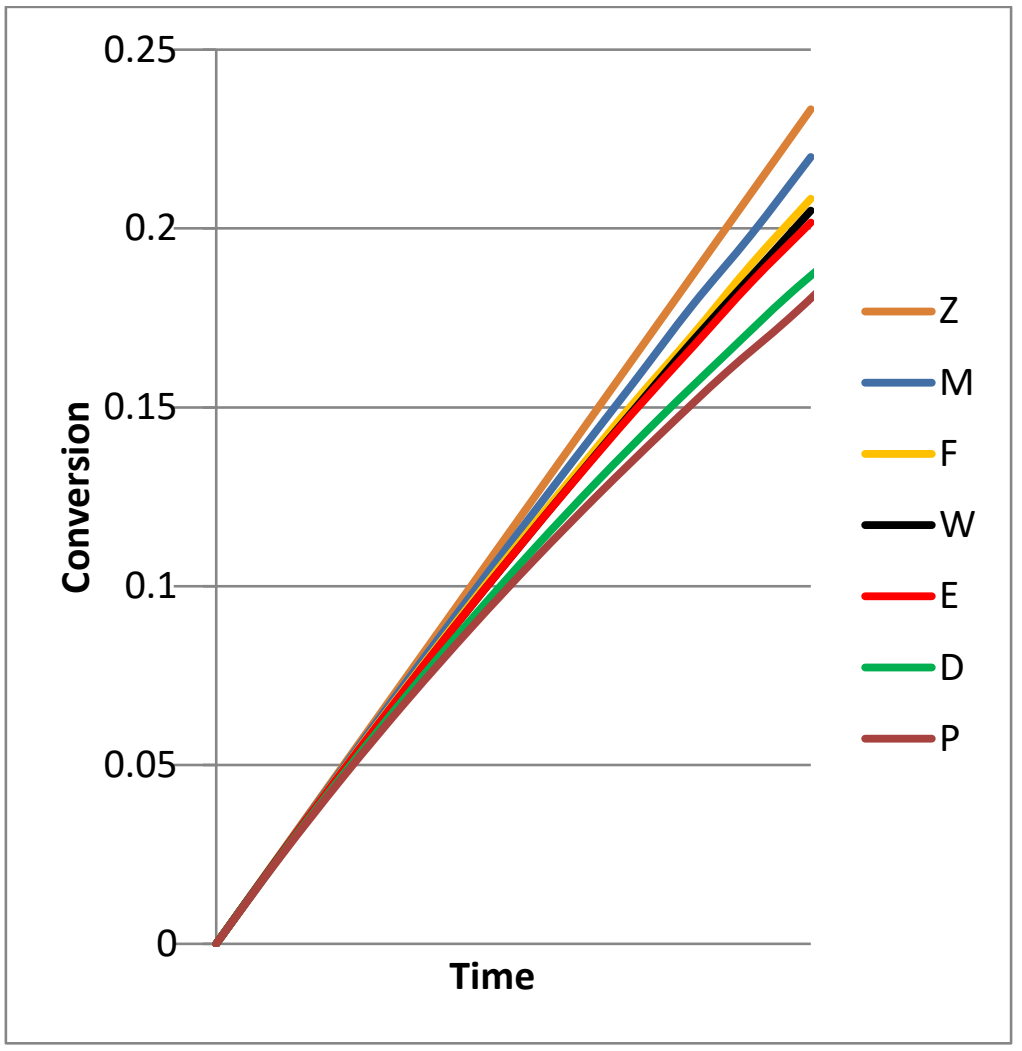

Fig. 4. Simulated progress curves before error addition. The equations used are identified by initial letter as for Zero order, Michaelis-Menten kinetics, First order, Weak product inhibition, Equilibrium approach, Deactivation of catalyst, and Product inhibition (stronger). See section 2.2 for details of the equations and parameters.

For each equation and parameter choice, progress curves were simulated with 4 different possibilities for error.

- High mixed error (4\% fractional and 0.0033 dimensionless absolute), sets $\mathrm{Zh}, \mathrm{Mh}$ etc

- Low mixed error (2\% fractional and 0.0017 dimensionless absolute), sets $\mathrm{Zl}$, Ml etc

- Pure absolute error (dimensionless value of 0.0033 ), sets Za, Ma etc

- Pure fractional error (4\%), sets Zf, Mf etc

Fig 1 above gives an indication of what these different error levels would mean for progress data. For each equation, parameter choice and error level, a dataset of 20 progress curves was simulated, and then analysed by fitting each of the 7 fitting models listed above. 


\subsubsection{Comparison of fitting to different curve models}

Fig $5 \mathrm{~A}$ shows how the accuracy of the fitted initial rate values depends on the models used both in data simulation and in fitting the resulting data sets. The datasets are presented in order of decreasing curvature in the error-free progress curves, from $P$ to $Z$ (see Fig. 4). As expected, the accuracy of the Linear fit (to conversions up to $10 \%)$ declines as the curvature increases $(p<0.01$ for bars 3 or more apart). As also expected, fit to a first order model is most accurate when the data has been simulated using the same model. Because the degree of curvature in the first order model is not adjustable, fits for datasets having greater curvature are considerably less accurate, and the RMS deviation also increases lower curvature, especially the linear $Z$ data ( $p<0.001$ for extreme cases versus central bars). The extent of curvature is also not adjustable for the second order model, and this also shows a clear optimum for accuracy with datasets $D(p<0.001$ compared with other bars), getting worse for greater (datasets $\mathrm{P}$ ) or lesser curvature. For fitting using the MichaelisMenten, Quadratic, Reverse Quadratic and Deactivating models, the accuracy also declines clearly as the curvature in the progress curve increases ( $p<0.001$ for datasets $P$ vs $Z$ ), even though these models can fit progress with different curvatures. These four models clearly perform better overall (RMS deviation over all 28 datasets 0.047 to 0.054 , compared with 0.080 for First Order, 0.109 for Second Order and 0.081 for Linear, $p \ll<$ $0.001)$.

Fig 5B shows how the size and type of error added to simulated datapoints affects the accuracy of initial rate estimates. It is clear that higher error makes the accuracy of a linear fit and all 4 adjustable curvature models worse, as might be expected. Other error conditions are rather similar, but absolute error may be more damaging than fractional contributions.

Based on these two figures the performance of the four fitting models offering adjustable curvature can be compared. The Michaelis-Menten model performs noticeably worse for the higher curvature data sets (Fig. 5A, $p<0.05$ vs Quadratic fit). For these data sets it generally fits with negative values of the curvature parameter, which are physically impossible in the underlying model, but still tends to underestimate the extent of curvature and the initial rate. The Reverse Quadratic model performs particularly poorly for high error conditions $(p<0.05)$. With these datasets it will sometimes fit a very strongly curved progress, with a greatly overestimated initial rate. These effects account for the slightly better overall performance of the Quadratic and Deactivation models, which probably show no significant differences. Interestingly the Deactivation model does not show clearly superior performance with datasets $D$, even though they were simulated using this model. These similarities may reflect the fact that the simple quadratic equation is a reasonable mathematical approximation to that for enzyme deactivation, when the curvature is not so large (see Supporting Information). 

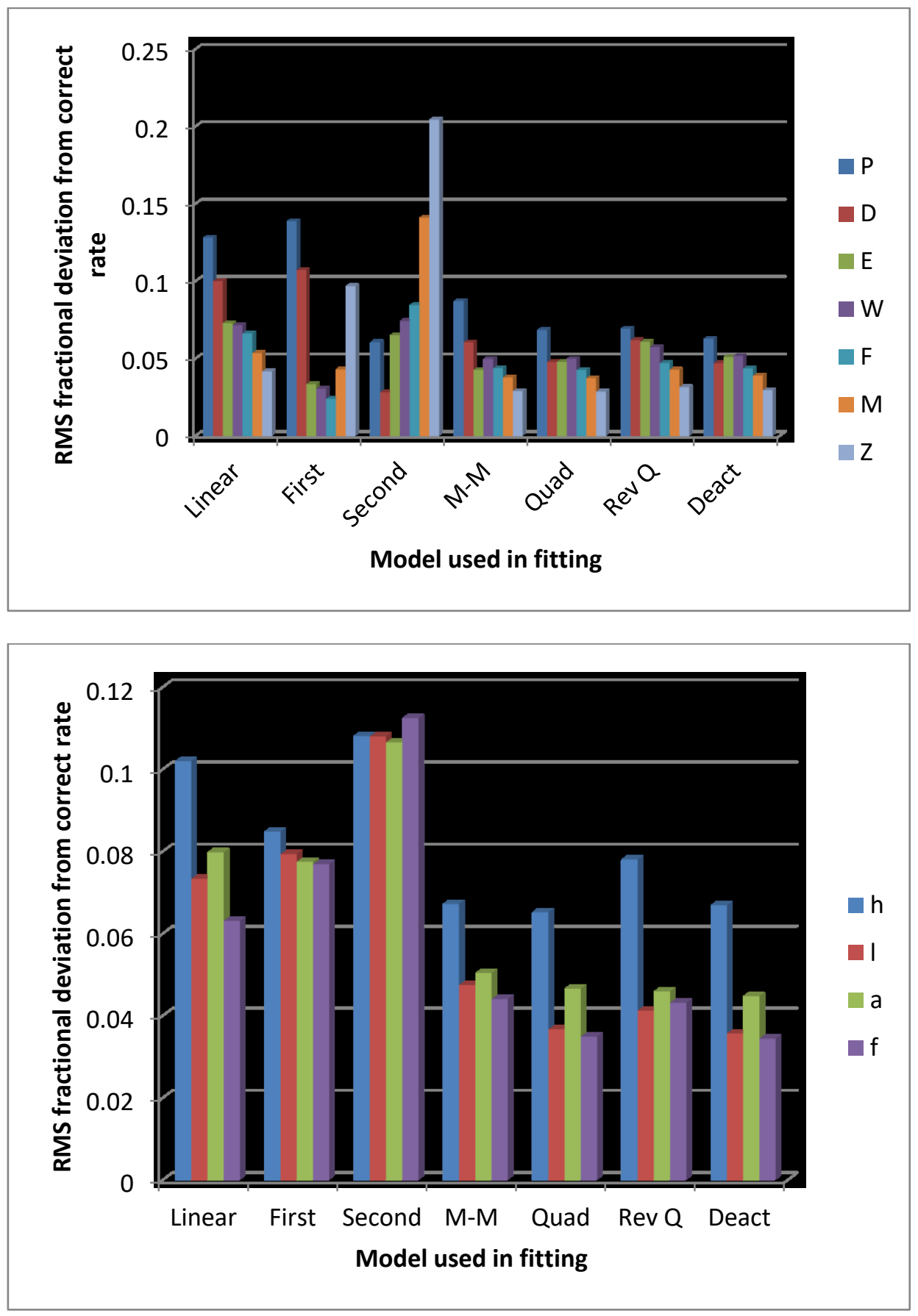

Figure 5. Accuracy of various fitting models. These are the RMS deviations of the relative initial rates fitted from the "true" value of 1 . Each time course had 11 equally spaced data points up to a conversion of about 0.18. Fitting of all models used the mixed error assumption. In part A each bar shows an overall value calculated from 4 datasets each of 20 time courses, simulated using the model equation shown, but with 4 different error conditions. The simulations are listed in order of decreasing curvature: P - product inhibition; D - catalyst deactivation; $\mathrm{E}$ - approach to equilibrium; $\mathrm{W}$ - weaker product inhibition; F - first order; $\mathrm{M}$ Michaelis-Menten kinetics; Z - Zero order, linear. In part B each bar shows an overall value for 6 datasets each of 20 time courses, simulated using the error pattern shown, but with 6 different model equations. The errors were: $\mathrm{h}$ - high mixed error; $\mathrm{I}$ - low mixed error; $\mathrm{a}$ - pure absolute error; $\mathrm{f}$ - pure fractional error. 


\subsubsection{Reproducibility of fitted rates}

As well as accuracy, it is desirable that fitted rates are also reproducible. This is particularly relevant when rates from different reactions are compared, or are to be used together to test kinetic models. So it is useful to examine whether fitting using different model equations produce wider or narrower ranges of rates from the same underlying simulation model.

Fig 6 A show how the mean and standard deviation of rates fitted using different models depends on the model used in simulating the data (i.e. probably on its extent of curvature). Rates from a Linear fit increase closer to the true value as the progress is less curved, as expected. The same increase is also seen for First and Second order fits, although for these models the initial rate is overestimated for the less curved progress. The overall increases, and even most of those between successive bars, are statistically significant $(p<0.001)$. The Michaelis-Menten fit noticeably underestimates the initial rate where the progress is most curved $(p<0.001$ compared with Quadratic fit), and this also happens to a lesser extent for Quadratic and Deactivation fits ( $p<$ 0.01 compared with less curved data). Standard deviations are not much affected by the extent of curvature in the data, but are significantly lower for the truly linear data $(p<0.01)$.

Fig 6B shows how the mean and standard deviation of fitted rates depends on the type of error in the simulated data. The variability (standard deviations) of initial rates are highest for the higher error incorporated in the simulations, as expected ( $p<0.001$ ). Also expected, the Linear fit (to conversion up to 0.1 ) almost always underestimates the true initial rate. The First and Second order fits generally give lower variability than other models $(p<<0.001$ ), although with the rate tending to be under- or over-estimated respectively. Because these models do not allow the degree of curvature to vary, they give a more reproducible fit. The models with adjustable curvature will sometimes produce a fit with too high curvature (and hence higher initial rate) or too low curvature (and hence lower initial rate). The First and Second order models avoid this, but at the expense of inaccurate rate estimates when the true curvature does not match the fixed level in the model. On average, the rates estimated by the Quadratic, Michaelis-Menten and Deactivating fits all tend to be rather lower than the correct value. The Reverse Quadratic fit tends to show higher variability (most $p<0.01$ ). This reflects its tendency with some datasets to produce very high curvature and hence a greatly overestimated initial rate. This effect also causes the model to give mean estimates closest to the true value. 

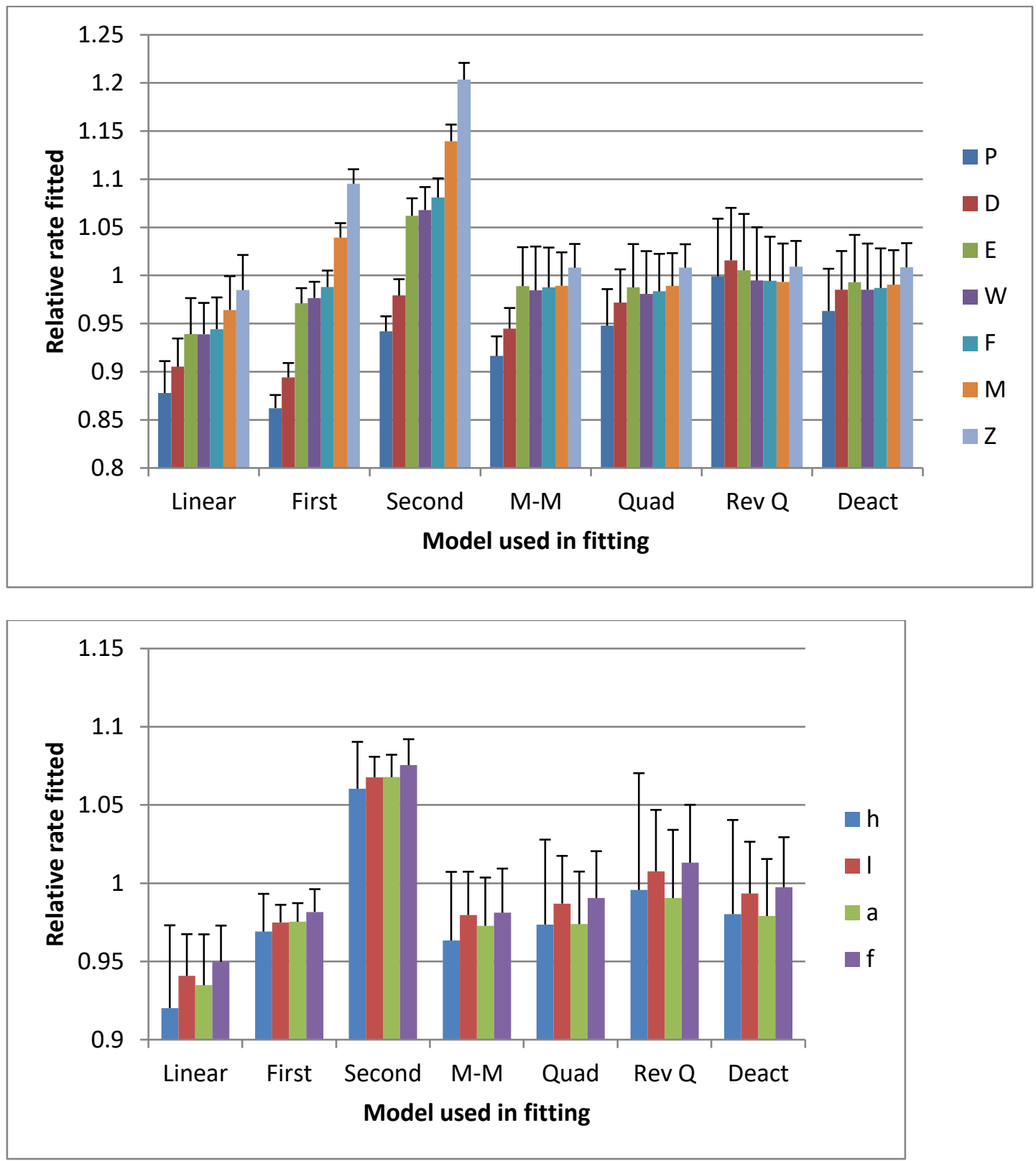

Fig 6. Mean and standard deviation of rates fitted by different models. The bars show the mean value relative to the "true" value of 1 , and the error bars indicate mean standard deviations. Fitting of all models used the mixed error assumption. A separate standard deviation was calculated for each dataset of 20 time courses which all had the same underlying progress - the value plotted is the mean of 4 or 6 such standard deviations. In part A each bar shows an overall value for 4 datasets each of 20 time courses, simulated using the model equation shown, but with 4 different error conditions. The simulations are listed in order of decreasing curvature: $\mathrm{P}$ - product inhibition; $\mathrm{D}$ - catalyst deactivation; $\mathrm{E}$ - approach to equilibrium; $\mathrm{W}$ weaker product inhibition; F - first order; M - Michaelis-Menten kinetics; Z - Zero order or fully linear. In part B each bar shows an overall value for 6 datasets each of 20 time courses, simulated using the error pattern shown, but with 6 different model equations. The errors were: $\mathrm{h}$ - high mixed error; I-low mixed error; $\mathrm{a}-$ pure absolute error; $f$ - pure fractional error. 


\title{
4.3.3. Further possible approaches to choosing fit equation
}

\author{
Select model fitting best
}

Although there is little to choose between the overall performance of the Quadratic, Deactivating, MichaelisMenten and Reverse Quadratic models, with each individual time course any one of them can give the most accurate initial rate estimate. One possibility investigated was to identify which of these models gave the best fit to each time course (as judged by lowest weighted sum of squares), and to select the rate fitted by this model. All 4 models were found among the selections, although the frequency tended to vary with the extent of curvature. Over all 28 datasets (560 time courses), the result was an overall RMS deviation of 0.048 , compared with 0.047 for the Quadratic or Deactivating models on their own. Hence it seems not sensible to use this more complicated approach, which also risks making individual rates less comparable when they are obtained by fits to different equations.

As is visible from Fig. 5, a first or second order fit can give accurate initial rate estimates when the extent of curvature in the data matches that of these models. Indeed in these cases they are more accurate than the more general curved models. Hence a test was made of switching between the First and Second models, selecting whichever gave the lowest weighted sum of squares for each time course. The resulting rates had an overall RMS accuracy (0.057) that was slightly worse than the general curve models. The approach had particular problems when there was the highest or no curvature (datasets $\mathrm{P}$ and $\mathrm{Z}$ ), where neither first nor second order fits could give an accurate initial rate. The method performed relatively well for datasets with high mixed error (RMS deviation 0.061) compared with 0.065 to 0.078 for the other 4 models. However, it is not recommended for use, because of the effect of switching between the two equations. If the second order fit is selected, with its greater curvature, this will result in a systematically higher rate estimate. For example, with a dataset of intermediate curvature (EI), rates of 1.046-1.080 were assigned in 6 cases where Second was selected, while 0.950-0.986 were assigned in 14 cases of First. Since a slight movement of a single point could change which model was selected, this would be very bad for comparability of reactions.

\section{Averaging fits to different models}

Another possible approach would be to take the rate as the average of those fitted by two or three of the general curve models. This has been tested for all possible combinations of the Quadratic, Reverse Quadratic and Deactivation models, but gave no significant change in overall RMS deviations.

\section{Errors may make "true" rates different}

Fig 7 shows that rates fitted to the same data using different model equations are quite strongly correlated with each other. This indicates that the random errors added to the data are responsible for much of their differences from the correct value of 1 , rather than the ability of the models to make a correct fit. For a given time course, random errors may tend to push several points above or below the error-free progress, so the rate is over- or under-estimated. Given this correlation, it might be argued that the fit to a model known to be correct for a given dataset is a basis to set the "true" rate. Since for this simulated data, we know which model is correct, the performance of other models can be tested on this basis.

For example, with datasets simulated using the deactivation model, RMS differences are calculated from the rate fitted using this same model (rather than from the actual rate used in simulation). On this basis the 
Quadratic model performs best, with RMS differences between 0.013 and 0.018 for 4 datasets, compared with 0.028-0.042 for Reverse Quadratic and 0.038-0.066 for Michaelis-Menten. However, this probably just reflects the similarity in fits between the Deactivation and Quadratic models, as discussed further in section 4.3.1. Comparing fits to datasets $F$, none of the general curve models agreed particularly well with the First Order model (which gives rather accurate initial rates for these datasets, Fig 5). RMS differences were 0.025 to 0.062 , with no model clearly superior -Reverse Quadratic was perhaps slightly worse overall. For datasets $\mathrm{Ml}$, Ma and Mf, fits to the Quadratic and Deactivation models agreed very well with the rate from Michaelis-Menten, with RMS differences 0.001-0.003. With the higher error dataset Mh, the RMS differences were 0.014 and 0.015. The Reverse Quadratic fits gave somewhat greater differences in every case (0.004 to 0.020 ).

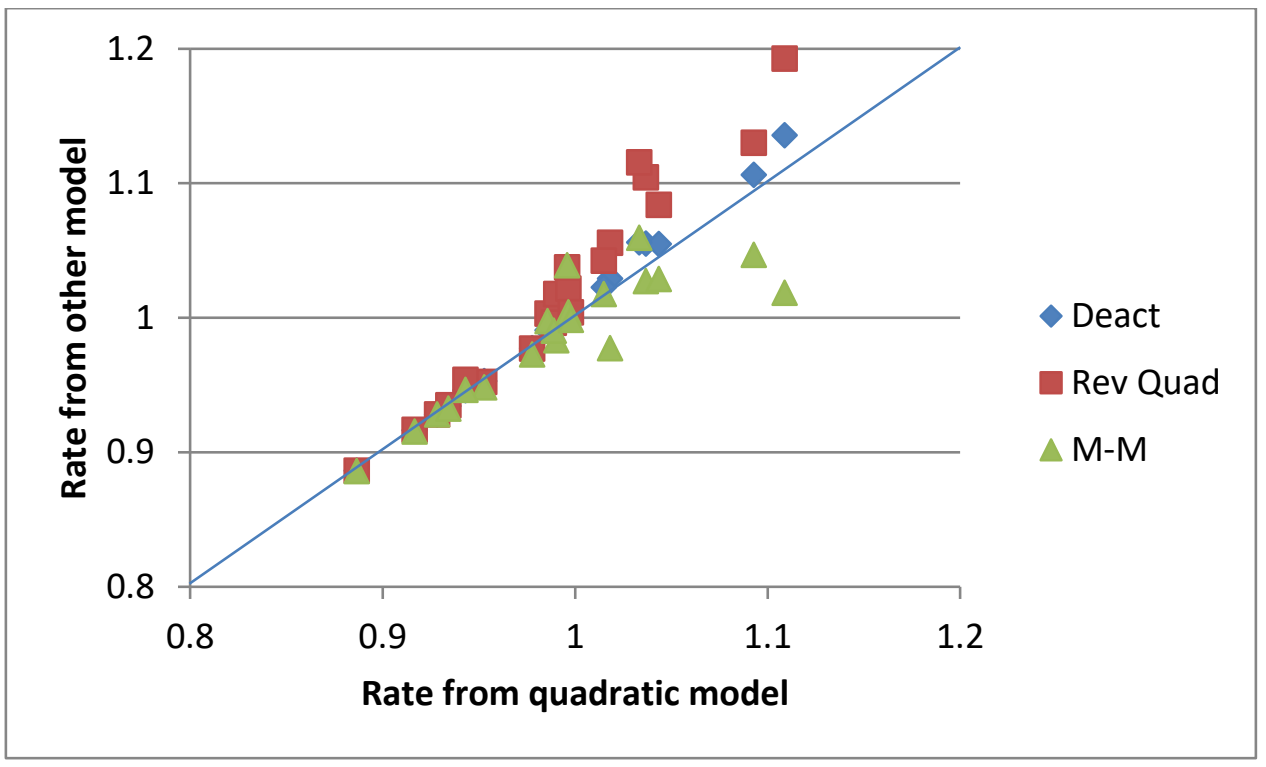

Fig. 7. Comparison of rates fitted to same data using different curve equations. Fits made to 20 time courses for medium curvature (Equilibrium approach model) and higher mixed error levels (dataset Eh). All fits used the mixed error assumption.

\section{Constraints on curvature parameters}

For models with adjustable curvature, more accurate rate estimates would result if the fitting algorithm were to reject excessively high curvature, by placing constraints on the value of the controlling parameter. But to do so would require a priori knowledge of the correct extent of curvature. With simulated datasets as used here, that would be possible. But in real experiments the available time course data are the only source of information about the true extent of curvature. Hence there is no empirical basis for saying that a particular time course is fitted with too great curvature. Consideration of the average behaviour of a series of time courses expected to have the same true curvature might be a route here. This is presented as an option to choose between linear and quadratic models in section 4.4.1.

\subsubsection{Effect of error assumption in fitting}


As noted above, in fitting equations to a progress curve it is necessary to make some assumption about the nature of the error in the points. Tests considered pure absolute error, pure fractional error, and mixed error such that the two contributions were equal for a conversion of 0.08. Fig 8 shows the results of these different assumptions, with datasets grouped as having the same type of error used in simulation. As can be seen, assuming pure fractional error in fitting produces the most accurate rate estimates when the actual error is purely fractional (right hand bars in Fig. $8, p<<0.001$ ). However, this assumption produces poorer rate estimates when the actual error is not purely fractional (most $p<0.001$ ), particularly when there is a high absolute component. The reasons for this are the same as in fitting straight lines, as detailed in section 4.2.4 above. Rather similar fits were usually found for assumptions of pure absolute or mixed error. As Fig 8 indicates, on average the mixed error assumption gives more accurate initial rate estimates $(p<0.05$ for low mixed or fractional error). It has been used for the results shown in previous sections.

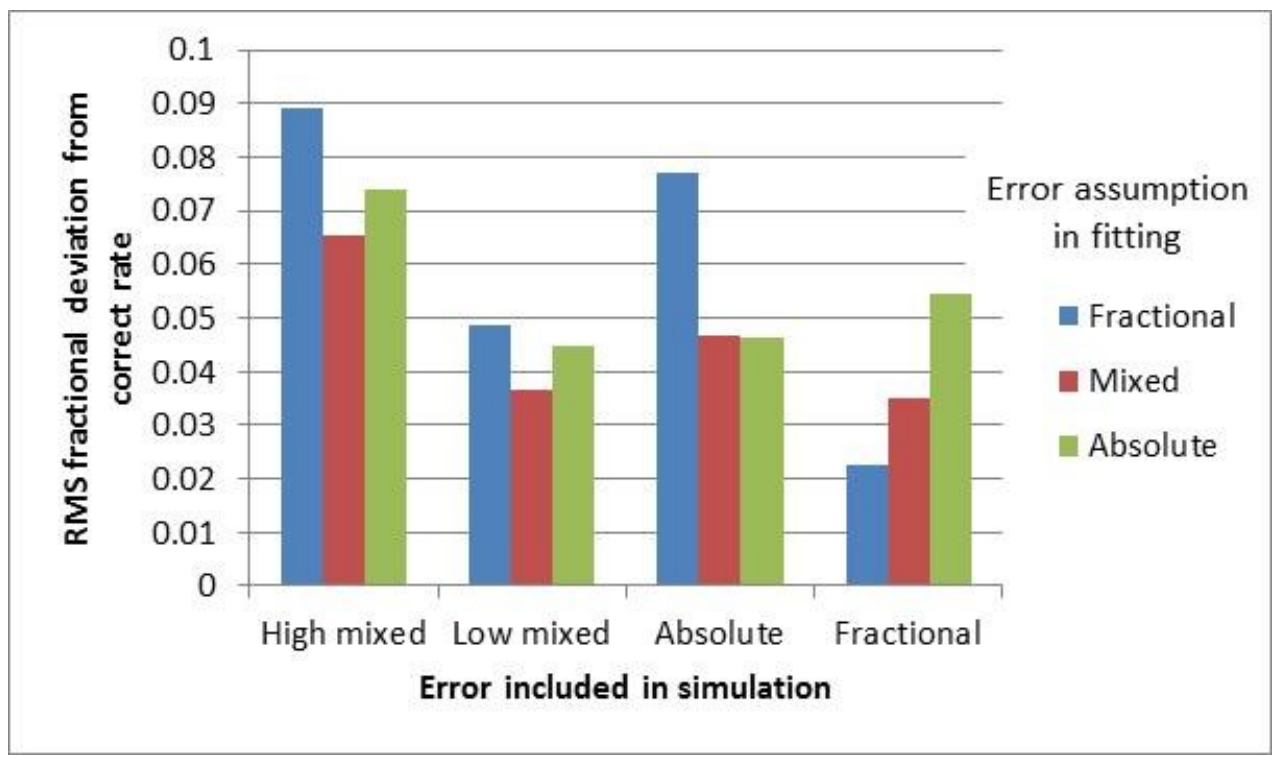

Fig. 8. Effect of type of error assumed in finding best fit curve. These are the RMS deviations from the "true" value of 1 of the relative initial rates fitted using the Quadratic model. Each bar shows an overall value for 7 datasets each of 20 time courses, simulated using the error pattern shown, but with 7 different underlying model equations. Rates were fitted by weighted least sum of squares, on the assumption that the error was either purely fractional, purely absolute or mixed. 


\subsection{Further options with linear and quadratic fits}

\subsubsection{Effects of number of time points used or available}

From Figures 5 and 6 above, it would seem that the quadratic fit is normally superior to a linear one. However, this is not really a fair comparison. Because the linear fit was restricted to points at a conversion less than 0.1, this would mean only 5 points were available, compared with 11 for the curve fits. So the linear and quadratic fits were tested for various numbers of points taken from datasets with a range of curvatures. In real experiments very often less than 11 points will be available. As expected, Fig 9A shows that the accuracy of the linear fit declines as more points (extending to longer times) are used, except in the case of truly linear progress (dataset $\mathrm{Zl}$ ). As reaction time increases the curvature becomes more and more significant, leading to an underestimate of initial rate from a linear fit. The accuracy is worse with the greater curvature of dataset $\mathrm{PI}$, and better with the smaller curvature of dataset MI. In contrast, the accuracy of the quadratic fit tends to decline as fewer data points are used, particularly for less than 7 (Fig 9A). With small numbers of datapoints, the random errors will sometimes make the quadratic fit see a much greater curvature than is really there, leading to a large overestimate of the initial rate (essentially a case of over-fitting with 3 adjustable parameters and as few as 4 data points). As noted above, the accuracy of the quadratic fit does tend to decline as the curvature of the progress increases, although the effect is much weaker than for a linear fit. As a result of these opposite trends, for the fairly low curvature of dataset Ml there is a clear cross-over in accuracy, with linear fit better if only the earlier points are used or available, but quadratic favoured for the full time course. Although results are displayed here only for one error type (lower mixed), a similar cross-over in accuracy was observed for datasets incorporating other error patterns (Supporting Information and available data files referenced therein). Fig. 9B shows these effects in another way. The linear fit always shows a lower standard deviation ( $p$ $<0.001$ ), which may make it preferable where reproducibility or comparability between reactions is more important than accuracy. As expected, reducing the number of data points increases the standard deviation for both types of fit ( $p<0.001$ for extreme points). For a curved progress, the rate estimated by linear fit declines steadily as more points extending to longer times are included, as the effect of curvature becomes more and more significant. For dataset $\mathrm{Pl}$ the mean rates estimated by linear and quadratic fits are always significantly different $(p<<0.001)$. 

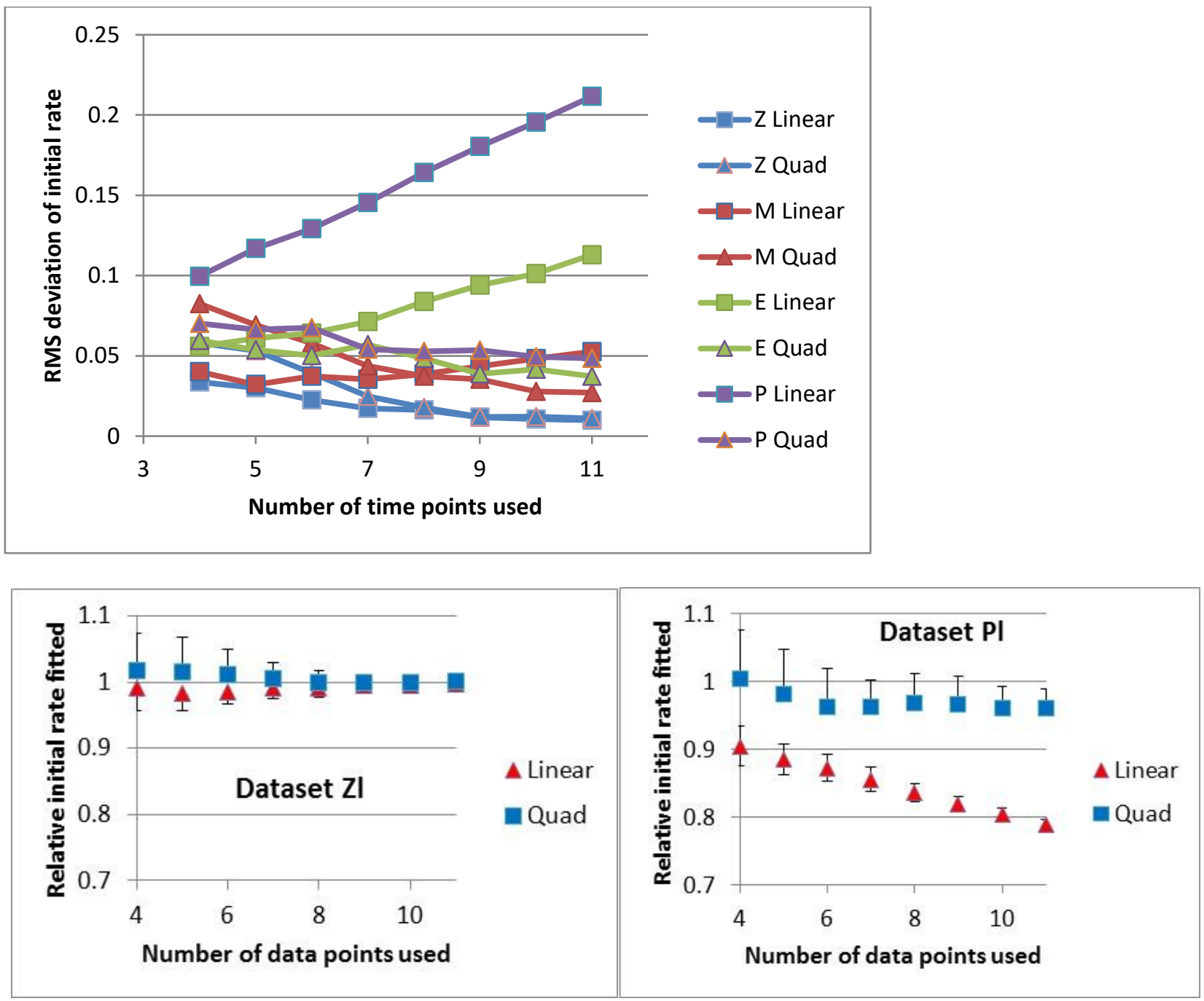

Fig 9. Comparison of quadratic and linear fits for different numbers of time points. The 20 time courses simulated with lower mixed error for each of zero order (ZI), Michaelis-Menten (MI), Equilibrium approach (EI) and Product inhibition (PI) were analysed. The linear and quadratic models were fitted to the first 4 up to the full 11 time points, using the mixed error assumption. Part A shows the RMS accuracy, while part B shows mean and standard deviation for the lowest and highest curvatures. Error bars show standard deviation, and are in only one direction where necessary to prevent overlap. RMS deviations differing by a ratio of more than 1.47 are significant at $p<0.05$, while a ratio of more than 2.11 gives $p<0.001$.

Based on the patterns in Fig 9, an attractive option might seem to be to use a quadratic fit when the progress is quite strongly curved, but switch to a linear one when curvature is slight or absent. But of course with real data there is no a priori method to know the extent of curvature, and this must be deduced from the data themselves. An obvious way to estimate the extent of curvature in the data is to fit a non-linear model such as 
the quadratic. Following a referee's suggestion, a test was made of selecting a linear or quadratic fit depending on which gave the lowest reduced chi square, i.e. weighted sum of squares / (number of time points - number of parameters fitted). However, for 4 data points the RMS deviation of the estimated rates was not significantly different from that for the quadratic fit (and thus worse than for the linear fit). For 11 points it was slightly worse than for a quadratic fit. The problem is that, as noted, random errors in the data will sometimes cause a quadratic fit to greatly over-estimate the extent of curvature, and as a result the initial rate. The quadratic may fit better and be selected, but actually the deviation from the correct initial rate will be less from a linear fit. The reduced chi-square is the accepted basis for model discrimination (deciding which model is most likely true). But the objective here is not to decide if the quadratic model better describes the data (it's probably not the true underlying model anyway), but whether it gives a better estimate of the true initial rate.

When a number of time courses expected to show similar degrees of curvature are available (e.g. replicates), looking at the whole set may allow a sensible judgment of how curved they are. With the quadratic fit, an indication of typical curvature is given by the average over the whole set of the coefficient $a$. Fig. 10 shows this value can clearly identify the increasing curvature as the model used goes from Zero order (Z) to MIchaelisMenten (M) to Equilibrium approach (E) to Product inhibition (P). (All Z and M bars are significantly smaller than all $E$ and $P$ ones, $p<0.01$ ). There is clearly still some scatter after averaging over 20 time courses, but a limit could be set that identifies those datasets where higher curvature makes a quadratic fit clearly superior to a linear one. An indication of the trend can also be seen by the frequency at which the quadratic fit gives a value of zero for the curvature coefficient (the algorithm does not allow a positive value). This defaulting to a linear fit occurred 45 times out of 80 time courses for model Z, 15 times for model M, once for model $E$ and not at all for model P.

Although this finding suggests a route to deciding when a quadratic fit is definitely to be preferred over a linear one, there are two remaining problems. First it requires a substantial number of time courses to average. This is easy enough in a simulation exercise, but may not be straightforward in an experimental study. Perhaps even more challenging is the requirement that all these time courses have similar curvature. They are unlikely all to be replicates, so human judgment is reintroduced with a priori assumptions about what experimental changes are unlikely to affect the extent of curvature. Many of the manipulations commonly studied in series of experiments, such as change in substrate or inhibitor concentrations, are quite likely to have effects on curvature. 


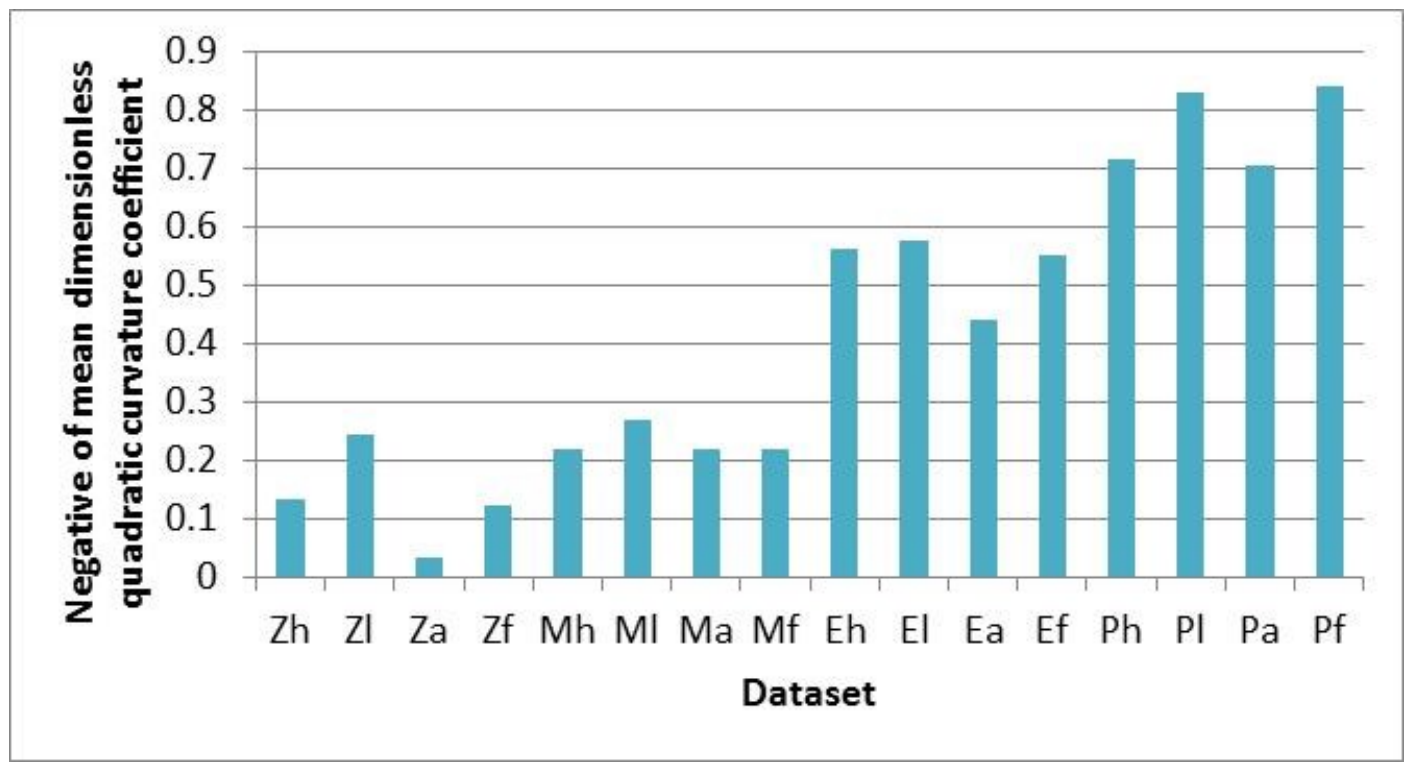

Fig. 10. Mean quadratic curvature parameter as a function of time course curvature. Datasets each of 20 timecourses were fitted to the quadratic model as for Fig. 5, using the mixed error assumption. The mean quadratic curvature control coefficient $(a)$ for the whole dataset was made dimensionless by multiplying by initial starting concentration and dividing by the square of initial rate. The dataset groups were based on the Zero order (Z), Michaelis-Menten (M), Equilibrium approach (E) and Product inhibition (P) models. For each model, errors could be higher mixed (h), lower mixed (I), purely absolute (a) or purely fractional (f). 


\subsubsection{Linear fit to selected initial points only}

The trends noted in the last section suggest that a useful approach might be to fit a straight line just to selected points at the start of the reaction progress, before curvature becomes too significant. As noted, the curvature would have to be detected from the data themselves. (Experience with the reaction might give an experimenter a view as to when curvature will become significant, but that will probably lead to an experimental design where samples will be restricted to times before this.)

A number of possible methods were tested to select initial data points to use for a linear fit. Each of these methods was tested against the datasets for Zero order, Michaelis-Menten, Equilibrium approach and Product inhibition, covering the range of curvature. All 4 error types were included for each underlying progress type. In each case a minimum number of points was set (usually the first 4), and straight lines were fitted to each possible initial selection of points (first 4 , first 5 , etc). The algorithm then chose the fit with the largest number of points before a termination condition was reached. In each case the termination condition introduces a parameter that controls the number of data points selected, and hence the initial rate estimated. Section 5 in Supporting Information shows the full range of methods tested, and the basis for selection of the optimal parameter value used in termination.

The following methods were selected for full testing:-

- " $v$ drop": termination when the fitted initial rate falls to a fraction of less than 0.99 of the previous value.

- "max v": select the highest fitted initial rate, subject to a minimum number of data points, set at 5.

- "sq dev rise"; termination when the mean weighted square deviation per data point increases by a factor of 1.02 or more, combined with a fall in rate

- "chisq rise": termination when the reduced chi-square value of the fit increase by a factor of 1.02 or more, combined with a fall in rate

Fig.11A shows that these algorithms selecting the number of initial points independently for each time course generally out-perform the Linear fit, which is always to the first 5 points $(p<0.01$ for most cases with datasets $Z, M$ and E). (They similarly out-perform a Linear fit to the first 4 points, see Supplementary Information.) None of the algorithms is clearly superior overall: their RMS deviations for all datasets combined are 0.060 ( $v$ drop), 0.066 (max v), 0.061 (sq dev rise) and 0.063 (chisq rise). The largest difference here is just significant at $p<$ 0.05. They are significantly different $(p<0.01)$ from 0.076 for Linear and 0.048 for Quad fits. For the lowest curvature "max $v$ " is probably superior ( $p<0.05$ compared with other algorithms). For the higher curvature, none of these modified linear algorithms can match the accuracy of a quadratic fit to all 11 time points. The Linear fit gives generally lower (less accurate) rate estimates than the point selection algorithms ( $<0.01$ for most comparisons; Fig 11B). The modified linear algorithms can choose to fit to more points for nearly linear progress, or to as few as 4 time points, reducing the effect of high curvature when this is present. Because the "max v" algorithm was restricted to a minimum of 5 time points (the optimal parameter chosen), it gives lower rate estimates for the highest curvature $(p<0.01)$. The max $v$ algorithm produces generally smaller standard deviations of the fitted rates ( $p<0.01$ for most comparisons; Fig 11B), compared with the other linear algorithms. On this basis it might be the best choice among the linear options, despite perhaps slightly lower overall accuracy. 

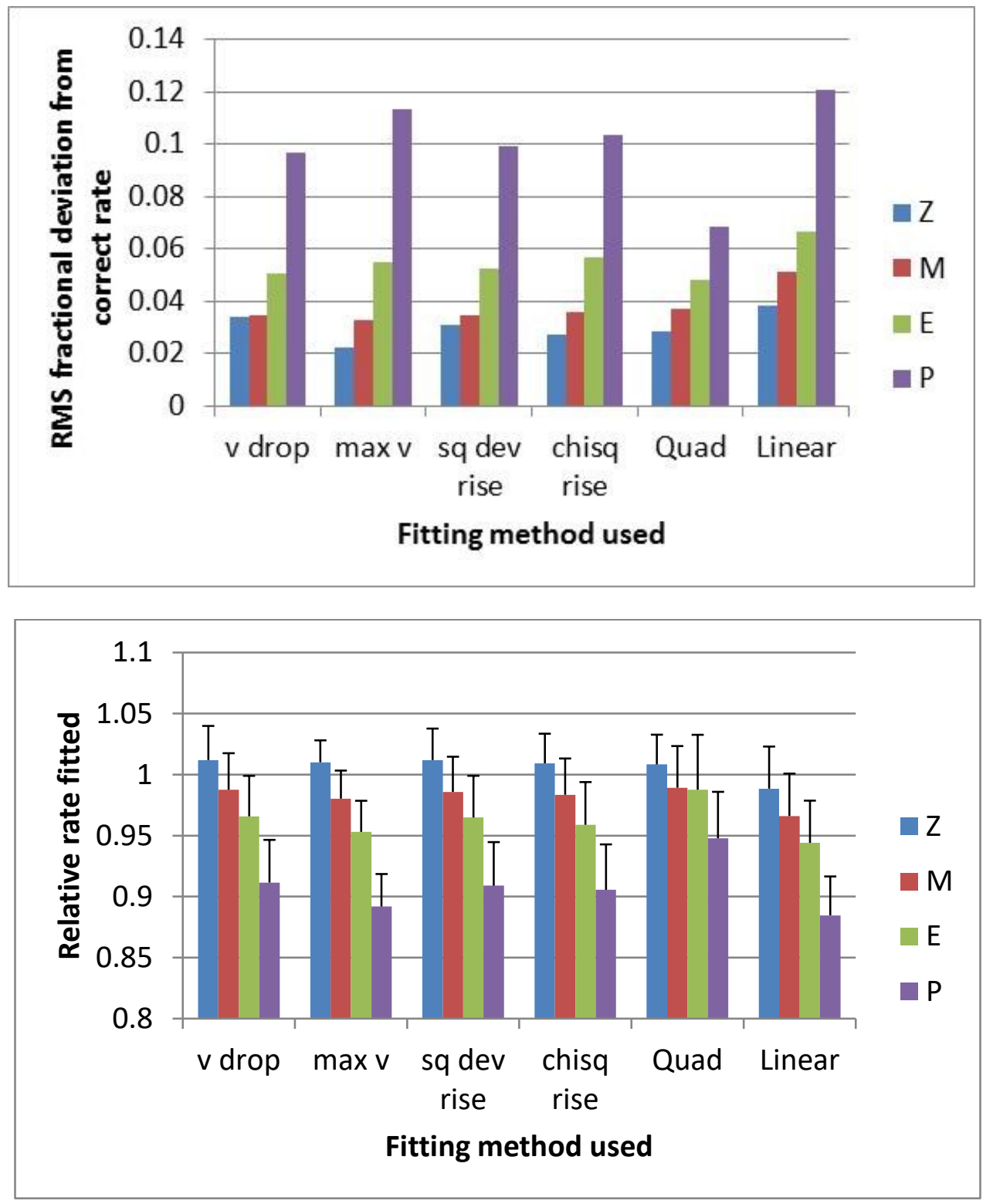

Fig. 11. Performance of various algorithms to select initial points for linear fit. Each bar shows an overall value calculated from 4 datasets each of 20 time courses, simulated using the model equation shown, but with 4 different error conditions. The simulations are listed in order of increasing curvature: Z - Zero order, linear; $\mathrm{M}$ - Michaelis-Menten kinetics; E - approach to equilibrium; P - product inhibition. As well as the point selection algorithms, for comparison is shown a Linear fit (to the first 5 points in each case for conversion $<0.1$ ) and a Quadratic fit to all 11 time points. Fitting of all models used the known error contributions used in simulation (hence the Quad and Linear fits are slightly different from those shown in previous figures). Part A shows the RMS deviations of the relative initial rates fitted from the "true" value of 1 . Part B shows the mean values, and the error bars indicate mean standard deviations. A separate standard deviation was calculated for each dataset of 20 time courses which all had the same underlying progress and error - the value plotted is the mean of 4 such standard deviations. 
As always, weighted least squares regression required error estimates for every data point. In this exercise the absolute and fractional error contributions were known from the simulations, and so were used. Of course, in a real experiment they will be uncertain, and estimates may often be very inaccurate. As noted, all regression fits depend to some extent on the relative size of fractional and absolute contributions to error, but not their absolute values. To examine the dependence on error estimates, the exercise was repeated using the default mixed error assumption as used in the curve fitting of section 4.3.1. The overall RMS deviations for the point selection algorithms increased, but only slightly by 1-2\% (Supplementary Information).

\subsection{General conclusions}

Based on the results presented, a number of general recommendations can be made. First there are features of the design of experiments

- It is obvious that reducing errors in data points will make initial rate estimate estimates more accurate. But this work shows how it is the absolute error contribution (rather than the fractional one) that is critical here, a less obvious point. With hindsight I can see why this is the case, but it had not occurred to me before getting these simulation results. So, minimise the absolute error contribution to the measurements, particularly where linear fits are to be used (sections 4.2.3 and 4.3.1). This can be affected by easily changed features of experimental design. For example, if samples are diluted before analysis, using smaller dilution factors for the early samples will probably reduce absolute error in product concentration measurements. If reactions are monitored by measurements of substrate depletion, the error in this will be effectively purely absolute throughout (as well as often higher). Hence measurement of product would be favoured wherever possible.

- Unless the absolute error contribution is extremely small, arrange sample times over a period such that the progress shows clear signs of curvature in at least some runs. At low conversion the effect of absolute error on accuracy of a linear fit outweighs that due to a little curvature (section 4.2.3). This is not a suggestion to use more sample times, where the gain in initial rate accuracy can be quite low (section 4.2.2) - rather samples should be spaced further apart instead of focussing on very small conversions.

When it comes to data analysis

- If there are less than 6 time points, a linear fit is favoured. Fitting a curve can sometimes give a very wrong estimate because of the effect of error in one or more points (section 4.4.1).

- Algorithms that select the number of points to include in a linear fit can out-perform using all the points where curvature is significant (section 4.4.2)

- For 9 or more data points, a simple quadratic fit is usually recommended, unless there are excellent a priori grounds for a theoretically based progress equation. In general the quadratic fit is at least as good as any other curve (sections 4.3.1 and 4.3.2).

- If reproducibility is more important than accuracy, a linear method may be favoured - perhaps where rates under a range of different conditions are to be compared with each other (section 4.4.1). 
These recommendations should be immediately useful to anyone estimating initial rates from discontinuous progress data. They do not amount to a completely objective set of rules that might be followed in all laboratories to produce fully consistent results. But any such proposed rules would have to come from a group of recognised international experts, not a single author.

\section{Disclosure statements}

No potential conflict of interest was reported by the author.

All the data that underlies this paper is available as supplemental online material and/or in the open access repository at https://pureportal.strath.ac.uk/.

\section{References}

Cornish-Bowden A (2012) Fundamentals of enzyme kinetics (4th rev. ed.). Wiley-Blackwell, Weinheim. Duggleby RG (1994) Analysis of progress curves for enzyme-catalyzed reactions - application to unstable enzymes, coupled reactions and transient-state kinetics. Biochim Biophys Acta 1205: 268-274. doi: 10.1016/0167-4838(94)90244-5

Golicnik M (2014) Progress-Curve Analysis Through Integrated Rate Equations and Its Use to Study Cholinesterase Reaction Dynamics. J Mol Neurosci 53: 330-334. doi: 10.1007/s12031-013-0129-y

Goudar CT, Sonnad JR, Duggleby RG (1999) Parameter estimation using a direct solution of the integrated Michaelis-Menten equation. Biochim Biophys Acta 1429: 377-383. doi: 10.1016/s0167-4838(98)002477

Liu BZ, Zhao YS, Zhao LN, Xie YL, Zhu S, Li ZR, Liu Y, Lu W, Yang XL, Xie GM, Zhong HS, Yu M, Liao H, Liao F (2009) An integration strategy to estimate the initial rates of enzyme reactions with much expanded linear ranges using uricases as models. Anal Chim Acta 631: 22-28. doi: 10.1016/j.aca.2008.10.021

Pinto MF, Ripoll-Rozada J, Ramos H, Watson EE, Franck C, Payne RJ, Saraiva L, Pereira PJB, Pastore A, Rocha F, Martins PM (2019) A simple linearization method unveils hidden enzymatic assay interferences. Biophys Chem 252: 106193-106193. doi: 10.1016/j.bpc.2019.106193

Poor J (1969) Interpolationsmethode zur Ermittlung der Anfangsgeschwindigkeit einer chemischen Reaktion mit nichtlinearem Verlauf. Chemicke Zvesti, 23(1): 73-76.

Press WH, Teukolsky SA, Vetterling WT, Flannery BP (1986) Numerical Recipes: The Art of Scientific Computing. Cambridge University Press, New York. 
Rakels JLL, Romein B, Straathof AJJ, \& Heijnen JJ (1994) Kinetic-analysis of enzymatic chiral resolution by progress curve evaluation. Biotechnol Bioeng 43: 411-422. doi: 10.1002/bit.260430509

Schnell S, Mendoza C (1997) Closed form solution for time-dependent enzyme kinetics. J Theor Biol 187: 207212. doi: 10.1006/jtbi.1997.0425

Soga N, Kimura K, Kinosita K, Yoshida M, Suzuki T (2017) Perfect chemomechanical coupling of FoF1-ATP synthase. Proc Nat Acad Sci USA 114: 4960-4965. doi: 10.1073/pnas.1700801114

Tang QX, Leyh TS (2010) Precise, Facile Initial Rate Measurements. J Phys Chem B 114: 16131-16136. doi: 10.1021/jp1055528

Varga Z, Kmecz I, Szecsenyi A, Szekely E (2017) Neat lipase-catalysed kinetic resolution of racemic 1phenylethanol and a straightforward modelling of the reaction. Biocatal Biotrans 35: 427-433. doi: 10.1080/10242422.2017.1360292 